\title{
Groups, group actions and fields definable in first-order topological structures
}

\author{
ROMAN WENCEL ${ }^{1}$ \\ Instytut Matematyczny Uniwersytetu Wrocławskiego
}

\begin{abstract}
Given a group $(G, \cdot), G \subseteq M^{m}$, definable in a first order structure $\mathcal{M}=(M, \ldots)$ equipped with a dimension function and a topology satisfying certain natural conditions, we find a large open definable subset $V \subseteq G$ and define a new topology $\tau$ on $G$ with which $(G, \cdot)$ becomes a topological group. Moreover, $\tau$ restricted to $V$ coincides with the topology of $V$ inherited from $M^{m}$. Likewise we topologize transitive group actions and fields definable in $\mathcal{M}$. These results require a series of preparatory facts concerning dimension functions, some of which might be of independent interest.
\end{abstract}

\section{$0 \quad$ Introduction}

A. Pillay in [Pi2] adapts Hrushovski's unpublished proof of a special case of Weil's 'group chunk theorem' [W] to show that a group definable in an o-minimal structure can be definably equipped with a topology making it a topological group and a definable manifold. The topology in question on a large definable subset of the considered definable group coincides with the usual topology induced by ordering of the structure. It follows that a field definable in an o-minimal structure can be definably made into a topological field.

As observed by A. Mosley in [Mo], Pillay's method works for groups and fields definable in sufficiently saturated first order topological structures (introduced in [Pi1]) on which certain very natural conditions have been imposed, like for example the exchange property of the algebraic closure. Among those topological structures we have several important classes of models satisfying various minimality conditions, namely: models of weakly o-minimal $/ C$-minimal theories in which acl has the exchange property as well as $P$-minimal structures. Both [Pi2] and [Mo] extensively use the theory of generics.

This paper contains an alternative approach towards the problem of topologization of definable algebraic objects. Instead of imposing the exchange property on acl, we consider topological structures equipped with so called dimension function.

Such a context is related to that of $[\mathrm{Mo}]$ but does not coincide with it. For example, it is easy too see that if $\mathcal{M}$ is a weakly o-minimal structure and for any $\mathcal{N} \succ \mathcal{M}$, acl has the exchange property, then the usual topological dimension (dim) is a dimension function in the sense of [vdD1]. On the other hand, if $\operatorname{dim}$ is a dimension function in a weakly o-minimal structure $\mathcal{M}$, then acl has the exchange property in $\mathcal{M}$ (see [We06] for details).

The paper is organized as follows. In $\S 1$ we fix some notation and discuss the basic notions that are used throughout the paper, like dimension functions and first order topological structures. In $\S 2$ we consider the issue of covering a group $G$ definable in a first order structure equipped with a (weak) dimension function by a finite number of its translates. We give effective bounds on the number of translates of a large definable subset of $G$ needed to cover $G$.

Some of the preparatory results proved in $\S 2$ are used in $\S 3-\S 5$ to investigate groups, group actions and fields definable in first order topological structures equipped with a dimension function. The key idea comes from the following fact.

\footnotetext{
${ }^{1}$ Research supported by the Polish Government grants N201 018 32/0800 and N N201 545938.
} 
Fact 0.1 [Pi2, Remark 1.14] Assume that $\mathcal{M}=(M, \leq, \ldots)$ is a sufficiently saturated o-minimal L-structure, $\varphi(\bar{x}, \bar{y}) \in L,|\bar{x}|=m,|\bar{y}|=n, \bar{b} \in M^{n}$ and $X \subseteq M^{m}$ is a non-empty definable set. The following conditions are equivalent.

(a) $\operatorname{dim}(X \backslash \varphi(M, \bar{b}))<\operatorname{dim}(X)$;

(b) $M \models \varphi(\bar{a}, \bar{b})$ whenever $\bar{a}$ is a generic of $X$ over $\bar{b}$.

Fact 0.1 makes it possible to transform the relevant proofs from [Pi2] so that every statement about a generic point satisfying certain property is replaced by its counterpart saying that the set of points with the property in question is large. In this way, modulo some dimension calculations, one obtains generics-free proofs of variants of Pillay's topologization results. An advantage of these modified proofs is that it is not necessary to work in a sufficiently saturated model.

\section{Preliminaries}

Let $\mathcal{M}$ be a first order structure whose underlying set is $M$. For $m \in \mathbb{N}_{+}$, by $D e f_{m}(\mathcal{M})$ we will denote the family of all subsets of $M^{m}$ that are definable in $\mathcal{M}$. We will also use the notation

$$
\operatorname{Def}(\mathcal{M}):=\bigcup_{m \in \mathbb{N}_{+}} \operatorname{Def} f_{m}(\mathcal{M})
$$

A function $d: \operatorname{Def}(\mathcal{M}) \longrightarrow \mathbb{N} \cup\{-\infty\}$ is said to be definable if for every $i \in \mathbb{N} \cup\{-\infty\}$ and any sets $X, Y, S \in \operatorname{Def}(\mathcal{M}), S \subseteq X \times Y$, each of the sets

$$
\left\{\bar{a} \in X: d\left(S_{\bar{a}}\right)=i\right\},\left\{\bar{b} \in Y: d\left(S^{\bar{b}}\right)=i\right\}
$$

is definable over the parameters needed to define $X, S$ and $Y, S$ respectively.

Following [vdD1], we will say that $d: \operatorname{Def}(\mathcal{M}) \longrightarrow \mathbb{N} \cup\{-\infty\}$ is a dimension function on $\mathcal{M}$ if the following conditions (A1)-(A6) are satisfied.

(A1) $\left(\forall m \in \mathbb{N}_{+}\right)\left(\forall X \in D e f_{m}(\mathcal{M})\right)(d(X)=-\infty \Longleftrightarrow X=\emptyset)$.

(A2) $(\forall a \in M)(d(\{a\})=0)$.

(A3) $d(M)=1$.

(A4) $\left(\forall m \in \mathbb{N}_{+}\right)\left(\forall X_{1}, X_{2} \in D e f_{m}(\mathcal{M})\right)\left(d\left(X_{1} \cup X_{2}\right)=\max \left\{d\left(X_{1}\right), d\left(X_{2}\right)\right\}\right)$.

(A5) $\left(\forall m \in \mathbb{N}_{+}\right)\left(\forall \sigma \in S_{m}\right)\left(\forall X \in D e f_{m}(\mathcal{M})\right)\left(d\left(X^{\sigma}\right)=d(X)\right)$, where $X^{\sigma}=\left\{\left\langle x_{\sigma(1)}, \ldots, x_{\sigma(m)}\right\rangle \in\right.$ $\left.M^{m}:\left\langle x_{1}, \ldots, x_{m}\right\rangle \in X\right\}$.

(A6) $\left(\forall m \in \mathbb{N}_{+}\right)\left(\forall X \in D e f_{m+1}(\mathcal{M})\right)(\forall i \in\{0,1\})(d(\{\langle\bar{x}, y\rangle \in X: \bar{x} \in X(i)\})=d(X(i))+i)$, where $X(i):=\left\{\bar{a} \in M^{m}: d\left(X_{\bar{a}}\right)=i\right\}$ is definable for $i=0,1$ over parameters needed to define $X$.

A function $d: \operatorname{Def}(\mathcal{M}) \longrightarrow \mathbb{N} \cup\{-\infty\}$ satisfying (A1)-(A5) will be called a weak dimension function on $\mathcal{M}$. As shown in [vdD1], a weak dimension function on $\mathcal{M}$ satisfies (A6) iff it is definable and the following condition (called addition property) holds:

$$
\left(\forall m, n \in \mathbb{N}_{+}\right)\left(\forall X \in D e f_{m+n}(\mathcal{M})\right)(\forall i \leq m)(d(\{\langle\bar{x}, \bar{y}\rangle \in X: \bar{x} \in X(i)\})=d(X(i))+i) .
$$


In particular this means that dimension functions are definable.

As in (A6) we care about parameters over which $X(i)$ is definable, our definition of dimension function slightly differs from that od L. van den Dries.

Assume that $d: \operatorname{Def}(\mathcal{M}) \longrightarrow \mathbb{N} \cup\{-\infty\}, m \in \mathbb{N}_{+}$and $X, Y \in \operatorname{De} f_{m}(\mathcal{M})$. We say that the set $X$ is $d$-large in $Y$ if $d(Y \backslash X)<d(Y)$. It is easy to see that a weak dimension function $d$ on $\mathcal{M}$ satisfies (A6) iff for any $m \in \mathbb{N}_{+}$and any $X \in D e f_{m+1}(X)$, if $\pi$ denotes the projection dropping the last coordinate, then the set $X(0)=\left\{\bar{a} \in \pi[X]: d\left(X_{\bar{a}}\right)=0\right\}$ is definable over the parameters needed to define $X$ and $d(X)=d(\pi[X])$ iff $X(0)$ is $d$-large in $\pi[X]$.

Suppose now that $d: \operatorname{Def}(\mathcal{M}) \longrightarrow \mathbb{N} \cup\{-\infty\}$ is a dimension function. Then $d$ is invariant under injective definable maps and satisfies the product rule, meaning that whenever $X, Y \in \operatorname{Def}(\mathcal{M})$ then $d(X \times Y)=d(X)+d(Y)$. If $X, Y \in \operatorname{Def}(\mathcal{M})$ and $f: X \longrightarrow Y$ is a definable function, then for every $k \in \mathbb{N} \cup\{-\infty\}$, the set $\left\{\bar{a} \in Y: d\left(f^{-1}[\bar{a}]\right)=k\right\}$ is definable over the parameters needed to define $X, Y$ and

$$
d\left(\bigcup\left\{f^{-1}[\bar{a}]: \bar{a} \in Y, d\left(f^{-1}[\bar{a}]\right)=k\right\}\right)=k+d\left(\left\{\bar{a} \in Y: d\left(f^{-1}[\bar{a}]\right)=k\right\}\right) .
$$

In particular $d(f[X]) \leq d(X)$. One may also easily show that whenever $n \geq 2, X \in \operatorname{De} f_{n}(\mathcal{M})$ and $d(X)<n$, then there is a projection $\pi: M^{n} \longrightarrow M^{n-1}$ such that $d(X)=d(\pi[X])$.

We say that $d: \operatorname{Def}(\mathcal{M}) \longrightarrow \mathbb{N} \cup\{-\infty\}$ is monotone if for any $X, Y \in D e f_{m}(\mathcal{M}), X \subseteq Y$ implies that $d(X) \leq d(Y)$. Obviously, $d$ satisfying (A4) is monotone.

The usual topological dimension in o-minimal structures is a dimension function (see [vdD2, Chapter 4]). In case $\mathcal{M}$ is a weakly o-minimal structure, the usual topological dimension on $\mathcal{M}$ is only a definable weak dimension function invariant under injective definable maps and satisfying the product rule (see [MMS] and [We06]). Moreover, a set definable in $\mathcal{M}$ has dimension 0 iff it is non-empty and finite.

Although in the weakly o-minimal context in general we cannot expect the topological dimension to have the addition property, two important weaker conditions (defined below) called weak addition properties of the first and of the second kind are satisfied.

Definition 1.1 Assume that $\mathcal{M}$ is a first order structure, $d:$ Def $(\mathcal{M}) \longrightarrow \mathbb{N} \cup\{-\infty\}$ is definable and $f: \mathbb{N} \longrightarrow \mathbb{N}_{+}$.

(a) We say that $d$ has the weak addition property of the first kind iff for any $m, n \in \mathbb{N}_{+}$, any definable sets $X \subseteq M^{m}, Y \subseteq M^{n}$ and $S \subseteq X \times Y$, if $\left\{\bar{a} \in X: S_{\bar{a}}\right.$ is d-large in $\left.Y\right\}$ is d-large in $X$, then $S$ is d-large in $X \times Y$.

(b) We say that $d$ has the weak addition property of the second kind for a function $f$ iff for any $m, n \in \mathbb{N}_{+}$, any definable sets $X \subseteq M^{m}, Y \subseteq M^{n}$ and $S \subseteq X \times Y$, if $S$ is d-large in $X \times Y$, then the set $\left\{\left\langle\bar{a}_{1}, \ldots, \bar{a}_{f(d(Y))}\right\rangle \in X^{f(d(\bar{Y}))}: S_{\bar{a}_{1}} \cup \ldots \cup S_{\bar{a}_{f(d(Y))}}\right.$ is d-large in $\left.Y\right\}$ is d-large in $X^{f(d(Y))}$.

It is not difficult to see that dimension functions have the weak addition property of the first kind and the weak addition property of the second kind for any function $f: \mathbb{N} \longrightarrow \mathbb{N}_{+}$. On the other hand, if $f, g: \mathbb{N} \longrightarrow \mathbb{N}_{+}$are functions such that $(\forall n \in \mathbb{N})(f(n) \leq g(n)), d$ is definable, monotone and satisfies the product rule and the weak addition property of the second kind for $f$, then $d$ has the weak addition property of the second kind for $g$.

It was demonstrated in [We06] that the usual topological dimension in weakly o-minimal structures has the weak addition property of the first kind and the weak addition property of the second kind for the function $f(n)=2^{n}$. Moreover, the example at the end of $\S 3$ of [We06] shows that there exists a weakly o-minimal structure $\mathcal{M}$ in which the topological dimension does not have the weak addition property of the second kind for the identity function. 
In this paper we will investigate algebraic objects definable in first order structures equipped with a topology. An important class of such structures was isolated by A. Pillay in [Pi1] and investigated by L. Matthews in [Ma1] and [Ma2].

Definition 1.2 [Pi1] Assume that $\mathcal{M}=(M, \ldots)$ is a first order L-structure and $\varphi(x ; \bar{y}) \in L$, where $|\bar{y}|=n \in \mathbb{N}_{+}$. Then $M$ is said to be a first order topological structure with respect to $\varphi$ if the set $\left\{\varphi(M ; \bar{a}): \bar{a} \in M^{n}\right\}$ is a basis of a topology on $M$.

Note that every weakly o-minimal structure is a first order topological structure with respect to the formula $\varphi\left(x ; y_{1} y_{2}\right)=\left(y_{1}<x<y_{2}\right)$.

If $\mathcal{M}=(M, \ldots)$ is a first order topological structure with respect to some formula $\varphi$ and $\tau$ is the topology on $M$ determined by $\varphi$, then we can define the product topology on all cartesian powers of $M$. Clearly, if $X \subseteq M^{n}$ is a definable set, then the interior of $X$, the closure of $X$ and the boundary of $X$ are definable over the parameters needed to define $X$. Suppose that additionally $d: \operatorname{Def}(\mathcal{M}) \longrightarrow \mathbb{N} \cup\{-\infty\}$. We will say that $\mathcal{M}$ has the continuity property with respect to $d$ and the topology determined by $\varphi$ if for any set $A \subseteq M, A$-definable non-empty sets $X \subseteq Y \subseteq M^{n}$ satisfying $d(X)=d(Y)$ and any $A$-definable function $f: Y \longrightarrow M$, there exists an $A$-definable set $V \subseteq X, d$-large in $X$ and open in $Y$, such that the function $f$ restricted to $V$ is continuous. Theorem 2.11 from [We06] shows that weakly o-minimal structures have the continuity property with respect to the usual topological dimension and the topology determined by ordering.

Let $\mathcal{M}=(M, \ldots)$ be a first order topological structure with respect to some formula $\varphi$. Denote by $\tau$ the topology determined by $\varphi$. Following [Ma2] (see Definition 6.2), we say that a definable set $C \subseteq M^{n}$ is a cell if it is a singleton or for some $m \in \mathbb{N}_{+}, m \leq n$, there exists a projection $\pi: M^{n} \longrightarrow M^{m}$ such that $\pi[C]$ is $\tau$-open in $M^{m}$ and $\pi \uparrow C$ is a $\tau$-homeomorphism. In particular, $\tau$-open definable sets in $M^{n}$ for every $n \in \mathbb{N}_{+}$are cells. It is not difficult to see that if $\tau$ is $T_{2}$, then every cell is a finite Boolean combination of open sets definable over the parameters needed to define $C$. As in Definition 6.3 of [Ma2], we say that $\mathcal{M}$ has the cell decomposition property (CDP) if for any $A \subseteq M$, an $A$-definable set $X \subseteq M^{n}$ and any $A$-definable function $f: X \longrightarrow M$, there exists a partition of $X$ into $A$-definable cells $X_{1}, \ldots, X_{k}$ such that $f \uparrow X_{i}$ is continuous for $i=1, \ldots, k$. For example, o-minimal structures, $p$-adically closed fields and (by [We07]) weakly o-minimal non-valuational expansions of groups have the CDP. Note that if $\mathcal{M}$ has the CDP and $\tau$ is $T_{2}$, then every set definable in $\mathcal{M}$ is a finite Boolean combination of open sets.

Assume that $\mathcal{M}=(M, \ldots)$ is a first order topological structure with respect to some formula $\varphi$ and $d: \operatorname{Def}(\mathcal{M}) \longrightarrow \mathbb{N} \cup\{-\infty\}$ is a dimension function. If non-empty open definable subsets of $M$ have $d$-dimension 1 , then (using the addition property) we can easily show that non-empty open definable subsets of $M^{n}$ are of $d$-dimension $n$. If additionally $\mathcal{M}$ has the CDP, then for any non-empty definable set $X \subset M^{n}$ and any $k \in \mathbb{N}_{+}, d(X) \geq k$ iff $X$ contains a cell $C$ which projects onto an open subset of $M^{k}$. Consequently, $d(X) \geq k$ iff there is a projection $\pi: M^{n} \longrightarrow M^{k}$ such that $\pi[X]$ has non-empty interior.

\section{Covering groups with translations of large sets}

Suppose that $\mathcal{M}$ is a first order structure equipped with a (weak) dimension function $d, G$ is a group definable in $\mathcal{M}$ and $V \subseteq G$ is a set definable in $\mathcal{M}$ which is $d$-large in $G$. The goal of this section is to find effective bounds (depending on $d(G)$ and $d(G \backslash V)$ ) on the number of translates of $V$ sufficient to cover $G$, under various assumptions on $d$ and $\mathcal{M}$. Throughout the section we will refer to the conditions (A1)-(A6) defining the dimension function. Some results obtained here will be used in sections $3-5$. The following lemma is obvious. 
Lemma 2.1 Assume that $\mathcal{M}$ is a first order structure and $d:$ Def $(\mathcal{M}) \longrightarrow \mathbb{N} \cup\{-\infty\}$ is a function invariant under injective definable maps. If $(G, \cdot)$ is a group definable in $\mathcal{M}$ and $V \subseteq G$ is a definable set, d-large in $G$, then each of the sets $g \cdot V \cdot h$ and $g \cdot V^{-1} \cdot h$ is d-large in $G$ for any $g, h \in G$.

Lemma 2.2 Assume that $\mathcal{M}$ is a first order structure, $d: \operatorname{Def}(\mathcal{M}) \longrightarrow \mathbb{N} \cup\{-\infty\}$ is a function satisfying the product rule and invariant under injective definable maps, $(G, \cdot)$ is a group definable in $\mathcal{M}$ and $V, W$ are non-empty definable subsets of $G$. If $d(G)=n, d(W)=k$ and $d(G \backslash V)=l$, then

(a) each of the sets

$$
S_{1}:=\{\langle\bar{a}, \bar{b}\rangle \in G \times G: \bar{b} \in W \backslash(\bar{a} \cdot V)\}, S_{2}:=\{\langle\bar{a}, \bar{b}\rangle \in G \times G: \bar{b} \in W \backslash(V \cdot \bar{a})\}
$$

has d-dimension equal to $k+l$;

(b) each of the sets

$$
\begin{aligned}
& S_{1}^{\prime}:=\left(\bigcup_{\bar{a} \in G}\{\bar{a}\} \times(\bar{a} \cdot V)\right) \cap(G \times W)=\{\langle\bar{a}, \bar{b}\rangle \in G \times G: \bar{b} \in(\bar{a} \cdot V) \cap W\}, \\
& S_{2}^{\prime}:=\left(\bigcup_{\bar{a} \in G}\{\bar{a}\} \times(V \cdot \bar{a})\right) \cap(G \times W)=\{\langle\bar{a}, \bar{b}\rangle \in G \times G: \bar{b} \in(V \cdot \bar{a}) \cap W\}
\end{aligned}
$$

has d-dimension equal to $k+d(V)$;

(c) (under additional assumption that d satisfies (A4)) if $V$ is d-large in $G$, then each of the sets

$$
\bigcup_{\bar{a} \in G}\{\bar{a}\} \times(\bar{a} \cdot V), \bigcup_{\bar{a} \in G}\{\bar{a}\} \times(V \cdot \bar{a})
$$

is d-large in $G \times W$.

Proof. We will prove each part of the lemma for the first set only. Consider a definable bijection $f: G \times G \longrightarrow G \times G$ given by $f(\bar{a}, \bar{b})=\left\langle\bar{b}, \bar{a}^{-1} \cdot \bar{b}\right\rangle$.

(a) As $f\left[S_{1}\right]=W \times(G \backslash V)$, by our assumptions we have that

$$
d\left(S_{1}\right)=d\left(f\left[S_{1}\right]\right)=d(W)+d(G \backslash V)=k+l .
$$

(b) Note that $f\left[S_{1}^{\prime}\right]=W \times V$. Consequently,

$$
d\left(S_{1}^{\prime}\right)=d\left(f\left[S_{1}^{\prime}\right]\right)=d(W)+d(V)=k+d(V) .
$$

(c) Note that $(G \times W) \backslash S_{1}^{\prime}=S_{1}$. If $V$ is $d$-large in $G$, then $l<n$. By $(\mathrm{A} 4), d(V)=n$. Hence $d\left(S_{1}\right)=k+l<k+n=k+d(V)=d\left(S_{1}^{\prime}\right)$. This proves that the set $\bigcup_{\bar{a} \in G}\{\bar{a}\} \times(\bar{a} \cdot V)$ is $d$-large in $G \times W$.

Lemma 2.3 Assume that $\mathcal{M}$ is a first order structure and $d: \operatorname{Def}(\mathcal{M}) \longrightarrow \mathbb{N} \cup\{-\infty\}$ is a dimension function. If $(G, \cdot)$ is a group definable in $\mathcal{M}$ and $W, V \subseteq G$ are definable sets such that $W \neq \emptyset$ and $V$ is d-large in $G$, then each of the sets

$$
\{\bar{a} \in G: \bar{a} \cdot V \text { is d-large in } W\},\{\bar{a} \in G: V \cdot \bar{a} \text { is d-large in } W\}
$$

is d-large in $G$. 
Proof. Let $d(G)=n, d(W)=k$, and $d(G \backslash V)=l$. Our assumptions guarantee that $n \geq k \geq 0$ and $n>l$. The case $V=G$ is trivial, so let $V \neq G$ (i.e. $l \geq 0$ ). We will only show that the set $\{\bar{a} \in G: \bar{a} \cdot V$ is $d$-large in $W\}$ is $d$-large in $G$. Define

$$
S=\{\langle\bar{a}, \bar{b}\rangle \in G \times G: \bar{b} \in W \backslash(\bar{a} \cdot V)\} .
$$

For $\bar{a} \in G$ and $\bar{b} \in W$ we have

$$
\begin{aligned}
& S_{\bar{a}}:=\{\bar{b} \in G:\langle\bar{a}, \bar{b}\rangle \in S\}=W \backslash(\bar{a} \cdot V) \\
& S^{\bar{b}}:=\{\bar{a} \in G:\langle\bar{a}, \bar{b}\rangle \in S\}=G \backslash\left(\bar{b} \cdot V^{-1}\right)=\bar{b} \cdot(G \backslash V)^{-1} .
\end{aligned}
$$

Note that for $\bar{a} \in G, \bar{a} \cdot V$ is not $d$-large in $W$ iff $d\left(S_{\bar{a}}\right)=k$. Also $d\left(S^{\bar{b}}\right)=d(G \backslash V)=l<n$. Then

Suppose for a contradiction that the set $\{\bar{a} \in G: \bar{a} \cdot V$ is $d$-large in $W\}$ is not $d$-large in $G$.

$$
d(\{\bar{a} \in G: \bar{a} \cdot V \text { is not } d \text {-large in } W\})=n,
$$

which means that

$$
d\left(\left\{\bar{a} \in G: d\left(S_{\bar{a}}\right)=k\right\}\right)=n .
$$

By monotonicity of $d$ and the addition property we have that

$$
d(S) \geq d\left(\bigcup_{\left\{\bar{a} \in G: d\left(S_{\bar{a}}\right)=k\right\}}\{\bar{a}\} \times S_{\bar{a}}\right)=d\left(\left\{\bar{a} \in G: d\left(S_{\bar{a}}\right)=k\right\}\right)+k=n+k .
$$

On the other hand, Lemma 2.2(a) implies that $d(S)=k+l<k+n$, a contradiction.

Lemma 2.4 Assume that $\mathcal{M}$ is a first order structure and $d: \operatorname{Def}(\mathcal{M}) \longrightarrow \mathbb{N} \cup\{-\infty\}$ is a dimension function. If $(G, \cdot)$ is a group definable in $\mathcal{M}$ such that $d(G)=n$ and $V \subsetneq G$ is a definable set, d-large in $G$, then some $d(G \backslash V)+2$ left (right) translates of $V$ cover $G$.

Proof. We will only show that there are $d(G \backslash V)+2$ left translates of $V$ covering $G$. Let $k=d(G \backslash V)$. Using Lemma 2.3, we inductively find $\bar{a}_{0}, \ldots, \bar{a}_{k+1} \in G$ such that

$$
d\left(G \backslash\left(\bar{a}_{0} \cdot V \cup \ldots \cup \bar{a}_{i} \cdot V\right)\right)<k-i+1
$$

whenever $i \leq k+1$. For $i=k+1$ this means that $G=\bar{a}_{0} \cdot V \cup \ldots \cup \bar{a}_{k+1} \cdot V$.

Corollary 2.5 Assume that $\mathcal{M}$ is a first order structure and $d: \operatorname{Def}(\mathcal{M}) \longrightarrow \mathbb{N} \cup\{-\infty\}$ is a dimension function. If $(G, \cdot)$ is a group definable in $\mathcal{M}$ such that $d(G)=n$ and $V \subseteq G$ is a definable set, $d$-large in $G$, then some $n+1$ left (right) translates of $V$ cover $G$.

Remark 2.6 Assume that $\mathcal{M}$ is a first order structure and $d: \operatorname{Def}(\mathcal{M}) \longrightarrow \mathbb{N} \cup\{-\infty\}$ is a function satisfying (A1), (A4) and invariant under injective definable maps. Assume also that $(G, \cdot)$ is a group definable in $\mathcal{M}, W \subseteq G$ is a finite set and $V \subseteq G$ is a definable set, d-large in $G$. Then for some $\bar{b} \in G$,

$$
W \subseteq(\bar{b} \cdot V) \cap(V \cdot \bar{b}) \cap\left(\bar{b} \cdot V^{-1}\right) \cap\left(V^{-1} \cdot \bar{b}\right) .
$$

Proof. The above inclusion holds for all $\bar{b}$ from $\bigcap_{\bar{a} \in W}\left[(\bar{a} \cdot V) \cap(V \cdot \bar{a}) \cap\left(\bar{a} \cdot V^{-1}\right) \cap\left(V^{-1} \cdot \bar{a}\right)\right]$. 
Lemma 2.7 Assume that $\mathcal{M}$ is a first order structure, $d: \operatorname{Def}(\mathcal{M}) \longrightarrow \mathbb{N} \cup\{-\infty\}$ is definable and invariant under injective definable maps, satisfies (A1), (A4), the product rule and the weak addition property of the second kind for a function $f: \mathbb{N} \longrightarrow \mathbb{N}_{+}$. If $(G, \cdot)$ is a group definable in $\mathcal{M}, V \subseteq G$ is a definable set, d-large in $G$, and $W \subseteq G$ is a non-empty definable set with $d(W)=k<d(G)$. Then

(a) each of the sets

$$
\begin{aligned}
& \left\{\left\langle\bar{a}_{1}, \ldots, \bar{a}_{f(k)}\right\rangle \in G^{f(k)}: \bar{a}_{1} \cdot V \cup \ldots \cup \bar{a}_{f(k)} \cdot V \text { is d-large in } W\right\}, \\
& \left\{\left\langle\bar{a}_{1}, \ldots, \bar{a}_{f(k)}\right\rangle \in G^{f(k)}: V \cdot \bar{a}_{1} \cup \ldots \cup V \cdot \bar{a}_{f(k)} \text { is d-large in } W\right\}
\end{aligned}
$$

is d-large in $G^{f(k)}$.

(b) $W$ can be covered with some $\sum_{i=0}^{k} f(i)$ left (right) translates of $V$;

(c) $G$ can be covered with some $1+\sum_{i=0}^{d(G \backslash V)} f(i)$ left (right) translates of $V$ (meaning that the sum equals 0 in case $V=G$ ).

Proof. (a) Let $W \subseteq G$ be a definable set of $d$-dimension $k$. By Lemma 2.2(c), the set $S:=$ $\bigcup_{\bar{a} \in G}\{\bar{a}\} \times(\bar{a} \cdot V)$ is $d$-large in $G \times W$, so by assumption the first set is $d$-large in $G^{f(k)}$. For the second we reason in a similar way.

(b) We proceed inductively on $d(W)$. Suppose first that $W \subseteq G$ is a definable set with $d(W)=0$. By Lemma 2.2(c), the set $S:=\bigcup_{\bar{a} \in G}\{\bar{a}\} \times(\bar{a} \cdot V)$ is $d$-large in $G \times W$, so by assumption, there are $\bar{a}_{1}, \ldots, \bar{a}_{f(0)} \in G$ such that $S_{\bar{a}_{1}} \cup \ldots \cup S_{\bar{a}_{f(0)}}=W$. In other words, $\bar{a}_{1} \cdot V \cup \ldots \cup \bar{a}_{f(0)} \cdot V$ covers $W$. So the lemma holds for $d(W)=0$.

Assume now that $W \subseteq G$ is a definable set with $d(W)=l+1<d(G)$ and suppose that the Lemma holds for dimension $l$. Again, by Lemma 2.2(c), the set $\bigcup_{\bar{a} \in G}\{\bar{a}\} \times(\bar{a} \cdot V)$ is $d$-large in $G \times W$. Our assumption guarantees that there are tuples $\bar{a}_{1}, \ldots, \bar{a}_{f(l+1)} \in G$ such that the union $\bar{a}_{1} \cdot V \cup \ldots \cup \bar{a}_{f(l+1)} \cdot V$ is $d$-large in $W$. By inductive hypothesis, the set $W \backslash\left(\bar{a}_{1} \cdot V \cup \ldots \cup \bar{a}_{f(l+1)} \cdot V\right)$ can be covered with some $\sum_{i=0}^{l} f(i)$ left translates of $V$. Consequently, some $\sum_{i=0}^{l+1} f(i)$ left translates of $V$ cover $W$. For right translates the proof is similar.

(c) is an immediate consequence of (a).

The above lemma and Theorem 3.6 from [We06] imply that if $(G, \cdot)$ is a group definable in a weakly o-minimal structure and $V \subsetneq G$ is a definable set, large in $G$, then $V$ is generic. More precisely, $G$ can be covered with some $2^{\operatorname{dim}(G \backslash V)+1}$ left (right) translates of $V$.

The following lemma strengthens Lemma 2.7.

Lemma 2.8 Assume that $\mathcal{M}$ is a first order structure and $d: \operatorname{Def}(\mathcal{M}) \longrightarrow \mathbb{N} \cup\{-\infty\}$ is a definable function invariant under injective definable maps and satisfying (A1), (A4), the product rule, the weak addition property of the first kind and the weak addition property of the second kind for a function $f$. Assume also that $(G, \cdot)$ is a group definable in $\mathcal{M}$ and $V, W$ are definable subsets of $G, V$ is d-large in $G$ and $d(G)>d(W)=k \geq 0$. For $m \in \mathbb{N}_{+}$define

$$
\begin{aligned}
& L_{m}=\left\{\left\langle\bar{a}_{1}, \ldots, \bar{a}_{m}\right\rangle \in G^{m}: W \subseteq \bar{a}_{1} \cdot V \cup \ldots \cup \bar{a}_{m} \cdot V\right\} \\
& R_{m}=\left\{\left\langle\bar{a}_{1}, \ldots, \bar{a}_{m}\right\rangle \in G^{m}: W \subseteq V \cdot \bar{a}_{1} \cup \ldots \cup V \cdot \bar{a}_{m}\right\} .
\end{aligned}
$$


(a) For every $m \geq \sum_{i=0}^{k} f(i)$, the sets $L_{m}$ and $R_{m}$ are d-large in $G^{m}$.

(b) If $d$ satisfies the addition property, then for every $m \geq k+1$, the sets $L_{m}$ and $R_{m}$ are d-large in $G^{m}$.

Proof. For $l \in\{0, \ldots, k\}$, let $g(l)=\sum_{s=0}^{l} f(k-s)$. We will inductively show that for $l=0, \ldots, k$, the set

$$
X_{l}:=\left\{\left\langle\bar{a}_{1}, \ldots, \bar{a}_{g(l)}\right\rangle \in G^{g(l)}: d\left(W \backslash\left(\bar{a}_{1} \cdot V \cup \ldots \cup \bar{a}_{g(l)} \cdot V\right)\right)<k-l\right\}
$$

is $d$-large in $G^{g(l)}$. Note that for $l=0$ the claim holds by Lemma $2.7($ a). So let $0 \leq l<k$ and suppose that the set $X_{l}$ is $d$-large in $G^{g(l)}$. Again, by Lemma $2.7(\mathrm{a})$, for every $\left\langle\bar{a}_{1}, \ldots, \bar{a}_{g(l)}\right\rangle \in X_{l}$, the set of all tuples $\left\langle b_{1}, \ldots, b_{f(k-l-1)}\right\rangle \in G^{f(k-l-1)}$ for which

$$
d\left(W \backslash\left(\bar{a}_{1} \cdot V \cup \ldots \cup \bar{a}_{g(l)} \cdot V \cup \bar{b}_{1} \cdot V \cup \ldots \cup \bar{b}_{f(k-l-1)} \cdot V\right)\right)<k-l-1
$$

is $d$-large in $G^{f(k-l-1)}$. Hence, by the weak addition property of the first kind, the set of all tuples $\left\langle\bar{a}_{1}, \ldots, \bar{a}_{g(l+1)}\right\rangle \in G^{g(l+1)}$ for which $d\left(W \backslash\left(\bar{a}_{1} \cdot V \cup \ldots \cup \bar{a}_{g(l+1)} \cdot V\right)<k-l-1\right.$ is $d$-large in $G^{g(l+1)}$. This shows that $L_{f(0)+\ldots+f(k)}$ is $d$-large in $G^{f(0)+\ldots+f(k)}$. By the weak addition property of the first kind (or just by the product rule), the set $L_{m}$ is $d$-large in $G^{m}$ whenever $m \geq \sum_{i=0}^{k} f(i)$. In a similar manner we prove that $R_{m}$ is $d$-large in $G^{m}$ for $m \geq \sum_{i=0}^{k} f(i)$.

If the function $d$ satisfies the addition property, then it satisfies the weak addition property for the function $f$ identically equal to 1 . From this (b) follows.

Corollary 2.9 Assume that $\mathcal{M}$ is a first order structure and $d: \operatorname{Def}(\mathcal{M}) \longrightarrow \mathbb{N} \cup\{-\infty\}$ is a definable function invariant under injective definable maps and satisfying (A1), (A4), the product rule, the weak addition property of the first kind and the weak addition property of the second kind for a function $f$. Assume also that $(G, \cdot)$ is a group definable in $\mathcal{M}$ and $V \subseteq G$ is a definable set, $d$-large in $G$. For $m \in \mathbb{N}_{+}$define

$$
\begin{aligned}
& L_{m}=\left\{\left\langle\bar{a}_{1}, \ldots, \bar{a}_{m}\right\rangle \in G^{m}: \bar{a}_{1} \cdot V \cup \ldots \cup \bar{a}_{m} \cdot V=G\right\} \\
& R_{m}=\left\{\left\langle\bar{a}_{1}, \ldots, \bar{a}_{m}\right\rangle \in G^{m}: V \cdot \bar{a}_{1} \cup \ldots \cup V \cdot \bar{a}_{m}=G\right\} .
\end{aligned}
$$

For every $m \geq 1+\sum_{0 \leq i \leq d(G \backslash V)} f(i)$, the sets $L_{m}$ and $R_{m}$ are d-large in $G^{m}$. In particular, if $d$ is a dimension function, then for every $m \geq \max (1, d(G \backslash V)+2)$, the sets $L_{m}$ and $R_{m}$ are d-large in $G^{m}$.

Theorem 2.10 Assume that $\mathcal{M}=(M, \ldots)$ is a first order L-structure which is $(|L|+|A|)^{+}$. saturated for some $A \subseteq M$ and $d: \operatorname{Def}(\mathcal{M}) \longrightarrow \mathbb{N} \cup\{-\infty\}$ is a definable function invariant under injective definable maps and satisfying (A1), (A4), the product rule, the weak addition property of the first kind and the weak addition property of the second kind for a function $f$. Assume also that $(G, \cdot)$ is a group definable in $\mathcal{M}$ over $A$ and $X \subseteq G$ is an A-type-definable set such that all $A$-definable sets $V \subseteq G$ containing $X$ are d-large in $G$.

(a) $G$ can be covered with some $1+\sum_{i<d(G)} f(i)$ left (right) translates of $X$.

(b) If $d$ has the addition property in $\mathcal{M}$, then $G$ can be covered with $d(G)+1$ left (right) translates of $X$. 
Proof. We will prove part (a) only for left translates. Let $k=1+\sum_{i<d(G)} f(i)$. For every $A$-definable set $V \subseteq G$ containing $X$ let $H(V)$ be the following $A$-definable set:

$$
H(V):=\left\{\left\langle\bar{a}_{1}, \ldots, \bar{a}_{k}\right\rangle \in G^{k}: \bar{a}_{1} \cdot V \cup \ldots \cup \bar{a}_{k} \cdot V=G\right\} .
$$

By Lemma 2.8, all sets $H(V)$ are $d$-large in $G^{k}$. By $(|L|+|A|)^{+}$-saturatedness of $\mathcal{M}$, the set

$$
H:=\bigcap\{H(V): X \subseteq V \subseteq G \text { and } V \text { is } A \text {-definable }\}
$$

is non-empty. Fix $\left\langle\bar{a}_{1}, \ldots, \bar{a}_{k}\right\rangle \in H$. It is clear that $\bar{a}_{1} \cdot V \cup \ldots \cup \bar{a}_{k} \cdot V=G$ whenever $V \subseteq G$ is an $A$-definable set containing $X$. We claim that $\bar{a}_{1} \cdot X \cup \ldots \cup \bar{a}_{k} \cdot X=G$. Suppose for a contradiction that there exists $\bar{a} \in G \backslash\left(\bar{a}_{1} \cdot X \cup \ldots \cup \bar{a}_{k} \cdot X\right)$. This means that $\bar{a} \notin \bar{a}_{i} \cdot X$ for $i=1, \ldots, k$. So there are $A$-definable sets $V_{1}, \ldots, V_{k}$ such that $X \subseteq V_{i} \subseteq G$ for $i=1, \ldots, k$ and $\bar{a} \notin \bar{a}_{i} \cdot V_{i}$. Let $V=V_{1} \cap \ldots \cap V_{k}$. Clearly, $V$ is A-definable, $X \subseteq V \subseteq G$, and $\bar{a} \notin \bar{a}_{1} \cdot V \cup \ldots \cup \bar{a}_{k} \cdot V$. This contradicts our choice of $\left\langle\bar{a}_{1}, \ldots, \bar{a}_{k}\right\rangle$.

(b) is an immediate consequence of (a).

\section{Topologization of definable groups}

Lemma 3.1 Assume that $\mathcal{M}=(M, \ldots)$ is a first order structure and $d: \operatorname{Def}(\mathcal{M}) \longrightarrow \mathbb{N} \cup\{-\infty\}$ a function invariant under injective definable maps satisfying $(A 1)$ and $(A 4)$. If $(G, \cdot)$ is a group definable in $\mathcal{M}$ and $V \subseteq G$ is a definable set, d-large in $G$, then

$$
(\forall \bar{g} \in G)\left(\exists \bar{g}_{1}, \bar{g}_{2} \in V\right)\left(\bar{g}=\bar{g}_{1} \cdot \bar{g}_{2}\right) .
$$

Proof. Let $\bar{g} \in G$. By Lemma 2.1, the set $\bar{g} \cdot V^{-1}$ is $d$-large in $G$. (A4) implies that the intersection $V \cap \bar{g} \cdot V^{-1}$ is also $d$-large in $G$ and has $d$-dimension equal to $d(G)$. By (A1), $V \cap \bar{g} \cdot V^{-1} \neq \emptyset$. So take an arbitrary $\bar{g}_{1} \in V \cap \bar{g} \cdot V^{-1}$. Then $\bar{g}_{1}=\bar{g} \cdot \bar{g}_{2}^{-1}$ for some $\bar{g}_{2} \in V$. Hence $\bar{g}_{1} \cdot \bar{g}_{2}=\bar{g}$.

The proof of Lemma 3.1 in fact shows that for any $\bar{g} \in G$, the set $\left\{\bar{x} \in V: \bar{x}^{-1} \cdot \bar{g} \in V\right\}$ is $d$-large in $G$. Similarly one can show that the set $\left\{\bar{x} \in V: \bar{g} \cdot \bar{x}^{-1} \in V\right\}$ is $d$-large in $G$.

Lemma 3.2 Assume that $\mathcal{M}=(M, \ldots)$ is a first order structure and $d: \operatorname{Def}(\mathcal{M}) \longrightarrow \mathbb{N} \cup\{-\infty\}$ a function invariant under injective definable maps satisfying (A1) and the product rule. If $G$ is a group definable in $\mathcal{M}$, and $V \subseteq G$ is a definable set, $d$-large in $G$, then

$$
d(\{\langle\bar{a}, \bar{b}\rangle \in G \times G: \bar{a} \cdot \bar{b} \notin V\})=d(G)+d(G \backslash V) .
$$

In particular, the set $\{\langle\bar{a}, \bar{b}\rangle \in G \times G: \bar{a} \cdot \bar{b} \in V\}$ is d-large in $G \times G$.

Proof. The lemma is obvious when $V=G$. So assume that $V \neq G$ and let $X=\{\langle\bar{a}, \bar{b}\rangle \in G \times G$ : $\bar{a} \cdot \bar{b} \notin V\}$. Note that the function $f: X \longrightarrow G \times(G \backslash V)$ given by $f(\bar{a}, \bar{b})=\langle\bar{a}, \bar{a} \cdot \bar{b}\rangle$ is a definable bijection. Therefore our assumptions about $d$ imply that

$$
d(X)=d(G \times(G \backslash V))=d(G)+d(G \backslash V)<d(G)+d(G)=d(G \times G) .
$$


Lemma 3.3 Assume that $\mathcal{M}=(M, \ldots)$ is a first order structure and $d: \operatorname{Def}(\mathcal{M}) \longrightarrow \mathbb{N} \cup\{-\infty\}$ is a dimension function. If $G$ is a group definable in $\mathcal{M}, V \subseteq G$ is a definable set, d-large in $G$ and $Y \subseteq G \times G$ is a definable set, d-large in $G \times G$, then the sets

$$
\begin{aligned}
& W_{1}:=\{\bar{a} \in V:\{\bar{b} \in G:\langle\bar{b}, \bar{a}\rangle \in Y\} \text { is d-large in } G\} ; \\
& W_{2}:=\left\{\bar{a} \in V:\left\{\bar{b} \in G:\left\langle\bar{b}^{-1}, \bar{b} \cdot \bar{a}\right\rangle \in Y\right\} \text { is d-large in } G\right\} .
\end{aligned}
$$

are both d-large in $G$.

Proof. Suppose for a contradiction that $W_{1}$ is not $d$-large in $G$. Then $d\left(V \backslash W_{1}\right)=d(G)$. For $\bar{a} \in V \backslash W_{1}, d(\{\bar{b} \in G:\langle\bar{b}, \bar{a}\rangle \notin Y\})=d(G)$. Since

$$
(G \times G) \backslash Y=\bigcup_{\bar{a} \in G}(\{\bar{b} \in G:\langle\bar{b}, \bar{a}\rangle \notin Y\} \times\{\bar{a}\}) \supseteq \bigcup_{\bar{a} \in V \backslash W_{1}}(\{\bar{b} \in G:\langle\bar{b}, \bar{a}\rangle \notin Y\} \times\{\bar{a}\}),
$$

we get $d((G \times G) \backslash Y)=d(G \times G)$. This means that $Y$ is not $d$-large in $G \times G$, a contradiction.

To prove that $W_{2}$ is $d$-large in $G$, suppose for a contradiction that $d\left(G \backslash W_{2}\right)=d(G)$. Then also $d\left(V \backslash W_{2}\right)=d(G)$. Observe that

$$
\begin{aligned}
V \backslash W_{2} & =\left\{\bar{a} \in V:\left\{\bar{b} \in G:\left\langle\bar{b}^{-1}, \bar{b} \cdot \bar{a}\right\rangle \in Y\right\} \text { is not } d \text {-large in } G\right\}= \\
& =\left\{\bar{a} \in G: d\left(\left\{\bar{b} \in G:\left\langle\bar{b}^{-1}, \bar{b} \cdot \bar{a}\right\rangle \notin Y\right\}\right)=d(G)\right\}
\end{aligned}
$$

Denote the group operation by $f$ (i.e. $f(\bar{a}, \bar{b})=\bar{a} \cdot \bar{b}$ for $\bar{a}, \bar{b} \in G$ ). Since for every $\bar{a} \in G$, the map $\bar{x} \mapsto\left\langle\bar{x}^{-1}, \bar{x} \cdot \bar{a}\right\rangle$ is a definable bijection between $\left\{\bar{b} \in G:\left\langle\bar{b}^{-1}, \bar{b} \cdot \bar{a} \notin Y\right\rangle\right\}$ and $f^{-1}(\bar{a}) \backslash Y$, we get

$$
V \backslash W_{2}=\left\{\bar{a} \in V: d\left(f^{-1}(\bar{a}) \backslash Y\right)=d(G)\right\} .
$$

Now,

$$
(G \times G) \backslash Y=\bigcup_{\bar{a} \in G}\left(f^{-1}(\bar{a}) \backslash Y\right) \supseteq \bigcup_{\bar{a} \in V \backslash W_{2}}\left(f^{-1}(\bar{a}) \backslash Y\right) .
$$

Consequently,

$$
d(G \times G) \geq d((G \times G) \backslash Y) \geq d\left(V \backslash W_{2}\right)+d(G)=2 d(G)=d(G \times G),
$$

which contradicts the fact that $Y$ is $d$-large in $G \times G$.

It is interesting to note that the above lemma has a variant in case $d$ is a weak dimension function invariant under definable injections and satisfying the product rule together with the weak addition property of the second kind for some function $h$.

Lemma 3.4 Assume that $\mathcal{M}=(M, \ldots)$ is a first order structure and $d: \operatorname{Def}(\mathcal{M}) \longrightarrow \mathbb{N} \cup\{-\infty\}$ is a definable weak dimension function invariant under injective definable maps and satisfying the product rule and the weak addition property of the second kind for a function $h: \mathbb{N} \longrightarrow \mathbb{N}$. Assume also that $G$ is a group definable in $\mathcal{M}, V \subseteq G$ is a definable set, d-large in $G$ and $Y \subseteq G \times G$ is a definable set, $d$-large in $G \times G$.

(a) The set

$$
W_{1}:=\left\{\left\langle\bar{a}_{1}, \ldots, \bar{a}_{h(d(G))}\right\rangle \in V^{h(d(G))}: \bigcup_{i=1}^{h(d(G))}\{\bar{b} \in G:\langle\bar{b}, \bar{a}\rangle \in Y\} \text { is d-large in } G\right\}
$$


is d-large in $G^{h(d(G))}$.

(b) The set

$$
W_{2}:=\left\{\left\langle\bar{a}_{1}, \ldots, \bar{a}_{h(d(G))}\right\rangle \in V^{h(d(G))}: \bigcup_{i=1}^{h(d(G))}\left\{\bar{b} \in G:\left\langle\bar{b}^{-1}, \bar{b} \cdot \bar{a}\right\rangle \in Y\right\} \text { is d-large in } G\right\}
$$

is d-large in $G^{h(d(G))}$.

Proof. (a) The set $Y$ is $d$-large in $G \times G$, so the set

$$
W_{1}^{\prime}=\left\{\left\langle\bar{a}_{1}, \ldots, \bar{a}_{h(d(G))}\right\rangle \in G^{h(d(G))}: \bigcup_{i=1}^{h(d(G))} Y^{\bar{a}_{i}} \text { is } d \text {-large in } G\right\}
$$

is $d$-large in $G^{h(d(G))}$. As $W_{1}=W_{1}^{\prime} \cap V^{h(d(G))}$, the set $W_{1}$ is also $d$-large in $G^{h(d(G))}$.

(b) Denote by $g$ the bijection from $G \times G$ onto itself defined by the formula: $g(\bar{a}, \bar{b})=\left\langle\bar{b}^{-1}, \bar{b} \cdot \bar{a}\right\rangle$. The set $Y$ is $d$-large in $G \times G$, so the set

$$
W_{2}^{\prime}:=\left\{\left\langle\bar{a}_{1}, \ldots, \bar{a}_{h(d(G))}\right\rangle \in G^{h(d(G))}: \bigcup_{i=1}^{h(d(G))} g^{-1}[Y]_{\bar{a}_{i}}\right\}
$$

is $d$-large in $G^{h(d(G))}$. As $W_{2}=W_{2}^{\prime} \cap V^{h(d(G))}$, the set $W_{2}$ is also $d$-large in $G^{h(d(G))}$.

Theorem 3.5 Assume that $\mathcal{M}$ is a first order topological structure with respect to some formula $\varphi(x ; \bar{y}), d: \operatorname{Def}(\mathcal{M}) \longrightarrow \mathbb{N} \cup\{-\infty\}$ is a dimension function and $\mathcal{M}$ has the continuity property with respect to $d$ and the topology determined by $\varphi$. Assume also that $(G, \cdot)$ is a group definable over some $A \subseteq M$ in $\mathcal{M}$ with $G \subseteq M^{m}$ and $d(G)=n$. Then there exist an $A$-definable set $V \subseteq G$ and a topology $\tau$ on $G$ such that

(a) $(G, \cdot)$ with the topology $\tau$ is a topological group;

(b) $V$ is d-large in $G$ and $\tau$-open in $G$;

(c) the topology $\tau$ restricted to $V$ coincides with the product topology induced from $M^{m}$;

(d) some $n+1$ left (right) translates of $V$ cover $G$.

Proof. Let $(G, \cdot)$ be a group definable in $\mathcal{M}$ over $A$ with $G \subseteq M^{m}$ and $d(G)=n$. By assumption, there is an $A$-definable set $V_{0} \subseteq G$ which is open in $G$ (with respect to the topology determined by $\varphi), d$-large in $G$ and such that the inversion map restricted to $V_{0}$ is continuous. The set $V_{0} \cap V_{0}^{-1}$ is $A$-definable and $d$-large in $G$, so again, there is an $A$-definable set $V_{1} \subseteq V_{0} \cap V_{0}^{-1}$ such that $V_{1}$ is $d$-large in $G$ and open in $G$. Note that the inversion restricted to $V_{1}$ is a continuous map from $V_{1}$ into $V_{0}$. Denote the group operation - by $f$ (i.e. $f(\bar{a}, \bar{b})=\bar{a} \cdot \bar{b}$ whenever $\bar{a}, \bar{b} \in G$ ). By Lemma 3.2 , the set $f^{-1}\left[V_{0}\right]$ is $d$-large in $G \times G$. Hence also $\left(V_{0} \times V_{0}\right) \cap f^{-1}\left[V_{0}\right]$ is $d$-large in $G \times G$. As previously, there is an $A$-definable set $Y_{0} \subseteq\left(V_{0} \times V_{0}\right) \cap f^{-1}\left[V_{0}\right]$ such that $Y_{0}$ is open in $G \times G$, $d$-large in $G \times G$, and $f\left\lceil Y_{0}\right.$ is continuous. Now define:

$$
\begin{aligned}
& W_{1}=\left\{\bar{a} \in V_{1}:\left\{\bar{b} \in G:\langle\bar{b}, \bar{a}\rangle \in Y_{0}\right\} \text { is d-large in } G\right\} \\
& W_{2}=\left\{\bar{a} \in V_{1}:\left\{\bar{b} \in G:\left\langle\bar{b}^{-1}, \bar{b} \cdot \bar{a}\right\rangle \in Y_{0}\right\} \text { is d-large in } G\right\} .
\end{aligned}
$$

The sets $W_{1}$ and $W_{2}$ are both definable over $A$ and (by Lemma 3.3) $d$-large in $G$. 
The set $V_{1}^{\prime}:=W_{1} \cap W_{2}$ is $A$-definable and $d$-large in $G$. Again, one can find an $A$-definable set $V_{2} \subseteq V_{1}^{\prime}$ such that $V_{2}$ is open in $G$ and $d$-large in $G$. The set $V_{2}^{-1}$ is $A$-definable, open in $G$ and $d$-large in $G$. Hence the set $V:=V_{2} \cap V_{2}^{-1}$ is $A$-definable, open in $G$ and $d$-large in $G$. Moreover, $V=V^{-1}$. Similarly $V \times V$ is $A$-definable, open in $G \times G$ and $d$-large in $G \times G$. Let

$$
Y=(V \times V) \cap\left\{\langle\bar{x}, \bar{y}\rangle \in Y_{0}: \bar{x} \cdot \bar{y} \in V\right\}=(V \times V) \cap Y_{0} \cap f^{-1}[V] .
$$

The set $Y$ is $A$-definable, open in $G \times G$ and $d$-large in $G \times G$. We know that for every $\bar{a} \in V$, each of the sets:

$$
\left\{\bar{b} \in G:\langle\bar{b}, \bar{a}\rangle \in Y_{0}\right\},\left\{\bar{b} \in G:\left\langle\bar{b}^{-1}, \bar{b} \cdot \bar{a}\right\rangle \in Y_{0}\right\}
$$

is $d$-large in $G$. Since for $\bar{a} \in V$ we have:

$$
\begin{aligned}
& \{\bar{b} \in G:\langle\bar{b}, \bar{a}\rangle \in Y\}=V \cap V \bar{a}^{-1} \cap\left\{\bar{b} \in G:\langle\bar{b}, \bar{a}\rangle \in Y_{0}\right\} \text { and } \\
& \left\{\bar{b} \in G:\left\langle\bar{b}^{-1}, \bar{b} \cdot \bar{a}\right\rangle \in Y\right\}=V \cap V \bar{a}^{-1} \cap\left\{\bar{b} \in G:\left\langle\bar{b}^{-1}, \bar{b} \cdot \bar{a}\right\rangle \in Y_{0}\right\},
\end{aligned}
$$

also each of the sets

$$
\{\bar{b} \in G:\langle\bar{b}, \bar{a}\rangle \in Y\},\left\{\bar{b} \in G:\left\langle\bar{b}^{-1}, \bar{b} \cdot \bar{a}\right\rangle \in Y\right\}
$$

is $d$-large in $G$ whenever $\bar{a} \in V$. Summing up, our reasoning shows that:

(a) the set $V$ is $A$-definable, open in $G$ and $d$-large in $G$;

(b) inversion is a continuous map from $V$ onto $V$;

(c) the set $Y \subseteq V \times V$ is $A$-definable, open in $G \times G$ and $d$-large in $G \times G$; moreover, the function $f \uparrow Y$ is continuous and assumes values in $V$;

(d) for every $\bar{a} \in V$, the sets $\{\bar{b} \in G:\langle\bar{b}, \bar{a}\rangle \in Y\},\left\{\bar{b} \in G:\left\langle\bar{b}^{-1}, \bar{b} \cdot \bar{a}\right\rangle \in Y\right\}$ are $d$-large in $G$; hence, for any $\bar{a} \in V$ and $\bar{a}^{\prime} \in G$, the set $\left\{\bar{b} \in G:\left\langle\bar{b} \cdot \bar{a}^{\prime}, \bar{a}\right\rangle \in Y\right\}$ is $d$-large in $G$;

(e) (by Corollary 2.9) there are $\bar{a}_{0}, \ldots, \bar{a}_{n} \in G$ such that

$$
\bar{a}_{0} \cdot V \cup \ldots \cup \bar{a}_{n} \cdot V=V \cdot \bar{a}_{0} \cup \ldots \cup V \cdot \bar{a}_{n}=G .
$$

Claim 1. For any $\bar{a}, \bar{b} \in G$, the set

$$
Z(\bar{a}, \bar{b}):=\{\bar{x} \in V: \bar{a} \cdot \bar{x} \cdot \bar{b} \in V\}
$$

is open in $V$ (and in $G$ ) and the map $\bar{x} \longmapsto \bar{a} \cdot \bar{x} \cdot \bar{b}$ is a homeomorphism (in $V$ ) from $Z(\bar{a}, \bar{b})$ onto $\bar{a} \cdot Z(\bar{a}, \bar{b}) \cdot \bar{b}$.

Proof of Claim 1. Let $\bar{a}, \bar{b} \in G$. We will show that for every $\bar{x}_{0} \in Z(\bar{a}, \bar{b})$, there exists a subset of $Z(\bar{a}, \bar{b})$ containing $\bar{x}_{0}$ which is open in $V$ and on which the map $\bar{x} \longmapsto \bar{a} \cdot \bar{x} \cdot \bar{b}$ is continuous. This is sufficient.

Fix $\bar{x}_{0} \in Z(\bar{a}, \bar{b})$. By Lemma 3.1, $\bar{b}=\bar{b}_{1} \cdot \bar{b}_{2}$ for some $\bar{b}_{1}, \bar{b}_{2} \in V$. (d) implies that the set

$$
Z_{0}:=\left\{\bar{c} \in V:\left\langle\bar{c} \cdot \bar{a}, \bar{x}_{0}\right\rangle \in Y,\left\langle\bar{c} \cdot \bar{a} \cdot \bar{x}_{0}, \bar{b}_{1}\right\rangle \in Y,\left\langle\bar{c} \cdot \bar{a} \cdot \bar{x}_{0} \cdot \bar{b}_{1}, \bar{b}_{2}\right\rangle \in Y,\left\langle\bar{c}^{-1}, \bar{c} \cdot \bar{a} \cdot \bar{x}_{0} \cdot \bar{b}\right\rangle \in Y\right\}
$$

is $d$-large in $G$. In particular $Z_{0} \neq \emptyset$. Fix $\bar{c} \in Z_{0}$ and let

$$
Z_{1}:=\left\{\bar{x} \in V:\langle\bar{c} \cdot \bar{a}, \bar{x}\rangle \in Y,\left\langle\bar{c} \cdot \bar{a} \cdot \bar{x}, \bar{b}_{1}\right\rangle \in Y,\left\langle\bar{c} \cdot \bar{a} \cdot \bar{x} \cdot \bar{b}_{1}, \bar{b}_{2}\right\rangle \in Y,\left\langle\bar{c}^{-1}, \bar{c} \cdot \bar{a} \cdot \bar{x} \cdot \bar{b}\right\rangle \in Y\right\} .
$$


Clearly, $\bar{x}_{0} \in Z_{1} \subseteq Z(\bar{a}, \bar{b})$. Note that

$$
\begin{aligned}
Z_{1}= & \left(f^{-1}\left[Y^{\bar{b}_{1}}\right] \cap Y\right)_{\bar{c} \cdot \bar{a}} \cap\left(f^{-1}\left[\left(f^{-1}\left[Y^{\bar{b}_{2}}\right] \cap Y\right)^{\bar{b}_{1}}\right] \cap Y\right)_{\bar{c} \cdot \bar{a}} \cap \\
& \left(f^{-1}\left[\left(f^{-1}\left[\left(f^{-1}\left[Y_{\bar{c}^{-1}}\right] \cap Y\right)^{\bar{b}_{2}}\right] \cap Y\right)^{\bar{b}_{1}}\right] \cap Y\right)_{\bar{c} \cdot \bar{a}} .
\end{aligned}
$$

Hence $Z_{1}$ is open in $V$. The function $\bar{x} \longmapsto \bar{a} \cdot \bar{x} \cdot \bar{b}$ restricted to $Z_{1}$ is a continuous map from $Z_{1}$ to $V$, because it is a composition of the continuous maps:

$$
\begin{aligned}
& \bar{x} \longmapsto\langle\bar{c} \cdot \bar{a}, \bar{x}\rangle \longmapsto \bar{c} \cdot \bar{a} \cdot \bar{x} \longmapsto\left\langle\bar{c} \cdot \bar{a} \cdot \bar{x}, \bar{b}_{1}\right\rangle \longmapsto \bar{c} \cdot \bar{a} \cdot \bar{x} \cdot \bar{b}_{1} \longmapsto \\
& \left\langle\bar{c} \cdot \bar{a} \cdot \bar{x} \cdot \bar{b}_{1}, \bar{b}_{2}\right\rangle \longmapsto \bar{c} \cdot \bar{a} \cdot \bar{x} \cdot \bar{b} \longmapsto\left\langle\bar{c}^{-1}, \bar{c} \cdot \bar{a} \cdot \bar{x} \cdot \bar{b}\right\rangle \longmapsto \bar{a} \cdot \bar{x} \cdot \bar{b} .
\end{aligned}
$$

This finishes the proof of Claim 1.

Claim 2. For any $\bar{a}, \bar{b} \in G$, the set

$$
Z^{\prime}(\bar{a}, \bar{b}):=\{\langle\bar{x}, \bar{y}\rangle \in V \times V: \bar{a} \cdot \bar{x} \cdot \bar{b} \cdot \bar{y} \in V\}
$$

is open in $V \times V$ and the function $\langle\bar{x}, \bar{y}\rangle \longmapsto \bar{a} \cdot \bar{x} \cdot \bar{b} \cdot \bar{y}$ is a continuous map from $Z^{\prime}(\bar{a}, \bar{b})$ to $V$.

Proof of Claim 2. Let $\bar{a}, \bar{b} \in G$. We will show that for every $\left\langle\bar{x}_{0}, \bar{y}_{0}\right\rangle \in Z^{\prime}(\bar{a}, \bar{b})$, there exists a subset of $Z^{\prime}(\bar{a}, \bar{b})$ containing $\left\langle\bar{x}_{0}, \bar{y}_{0}\right\rangle$ which is open in $V \times V$ and on which the map $\langle\bar{x}, \bar{y}\rangle \longmapsto \bar{a} \cdot \bar{x} \cdot \bar{b} \cdot \bar{y}$ is continuous. This is sufficient.

Fix $\left\langle\bar{x}_{0}, \bar{y}_{0}\right\rangle \in Z^{\prime}(\bar{a}, \bar{b})$. By Lemma 3.1, $\bar{b}=\bar{b}_{1} \cdot \bar{b}_{2}$ for some $\bar{b}_{1}, \bar{b}_{2} \in V$. As in the proof of Claim 1 , the set

$$
\begin{aligned}
Z_{0}^{\prime}:= & \left\{\bar{c} \in V:\left\langle\bar{c} \cdot \bar{a}, \bar{x}_{0}\right\rangle \in Y,\left\langle\bar{c} \cdot \bar{a} \cdot \bar{x}_{0}, \bar{b}_{1}\right\rangle \in Y,\right. \\
& \left.\left\langle\bar{c} \cdot \bar{a} \cdot \bar{x}_{0} \cdot \bar{b}_{1}, \bar{b}_{2}\right\rangle \in Y,\left\langle\bar{c} \cdot \bar{a} \cdot \bar{x}_{0} \cdot \bar{b}, \bar{y}_{0}\right\rangle \in Y,\left\langle\bar{c}{ }^{-1}, \bar{c} \cdot \bar{a} \cdot \bar{x}_{0} \cdot \bar{b} \cdot \bar{y}_{0}\right\rangle \in Y\right\}
\end{aligned}
$$

is $d$-large in $G$. In particular $Z_{0}^{\prime} \neq \emptyset$. Fix $\bar{c} \in Z_{0}^{\prime}$ and let

$$
\begin{aligned}
Z_{1}^{\prime}:= & \left\{\langle\bar{x}, \bar{y}\rangle \in V \times V:\langle\bar{c} \cdot \bar{a}, \bar{x}\rangle \in Y,\left\langle\bar{c} \cdot \bar{a} \cdot \bar{x}, \bar{b}_{1}\right\rangle \in Y,\right. \\
& \left.\left\langle\bar{c} \cdot \bar{a} \cdot \bar{x} \cdot \bar{b}_{1}, \bar{b}_{2}\right\rangle \in Y,\langle\bar{c} \cdot \bar{a} \cdot \bar{x} \cdot \bar{b}, \bar{y}\rangle \in Y,\left\langle\bar{c}^{-1}, \bar{c} \cdot \bar{a} \cdot \bar{x} \cdot \bar{b} \cdot \bar{y}\right\rangle \in Y\right\} .
\end{aligned}
$$

Note that like in Claim $1,\left\langle\bar{x}_{0}, \bar{y}_{0}\right\rangle \in Z_{1}^{\prime} \subseteq Z^{\prime}(\bar{a}, \bar{b}), Z_{1}^{\prime}$ is open in $V \times V$ and the function $\langle\bar{x}, \bar{y}\rangle \longmapsto \bar{a} \cdot \bar{x} \cdot \bar{b} \cdot \bar{y}$ restricted to $Z_{1}^{\prime}$ is a continuous map from $Z_{1}^{\prime}$ to $V$. This finishes the proof of Claim 2.

Now, define the topology $\tau$ on $G: Z \subseteq G$ is $\tau$-open iff for any $\bar{g} \in G,(\bar{g} \cdot Z) \cap V$ is open in $V$. Clearly, if $Z \subseteq V$ is definable in $\mathcal{M}$, then $Z$ is open in $G$ iff $Z$ is $\tau$-open. The product $G \times G$ is naturally equipped with the product $\tau$-topology, and it is easy to see that a definable set $Z \subseteq G \times G$ is $\tau$-open iff for any $\bar{g}_{1}, \bar{g}_{2} \in G,\left(\left\langle\bar{g}_{1}, \bar{g}_{2}\right\rangle \cdot Z\right) \cap(V \times V)$ is open in $V \times V$.

Claim 3. Inversion is a $\tau$-homeomorphism on $G$. Since

Proof of Claim 3. Let $W \subseteq G$ be a $\tau$-open set. We will be done if we prove that $W^{-1}$ is $\tau$-open.

$$
W^{-1}=\left(W \cap\left(\bar{a}_{0} \cdot V \cup \ldots \cup \bar{a}_{n} \cdot V\right)\right)^{-1}=\bigcup_{i \leq n}\left(W \cap\left(\bar{a}_{i} \cdot V\right)\right)^{-1},
$$


it is enough to show that $(W \cap(\bar{a} \cdot V))^{-1}$ is $\tau$-open whenever $\bar{a} \in G$. Let $\bar{a} \in G$ and $Z=W \cap(\bar{a} \cdot V)$. The set $W$ is $\tau$-open, so $\left(\bar{a}^{-1} \cdot W\right) \cap V=\bar{a}^{-1} \cdot Z$ is open in $V$. By (b), $\left(\bar{a}^{-1} \cdot Z\right)^{-1}=Z^{-1} \cdot \bar{a}$ is open in $V$. By Claim 1, if $\bar{g} \in G$, then $\left(\bar{g} \cdot\left(Z^{-1} \cdot \bar{a}\right) \cdot \bar{a}^{-1}\right) \cap V=\left(\bar{g} \cdot Z^{-1}\right) \cap V$ is open in $V$. So $Z^{-1}$ is $\tau$-open.

Claim 4. The group operation · is $\tau$-continuous on $G$.

Proof of Claim 4. Let $W \subseteq G$ be a $\tau$-open set. We must prove that the set $f^{-1}[W]$ is $\tau$-open in $G \times G$. Since

$$
f^{-1}[W]=f^{-1}\left[W \cap\left(\bar{a}_{0} \cdot V \cup \ldots \cup \bar{a}_{n} \cdot V\right)\right]=\bigcup_{i \leq n} f^{-1}\left[W \cap\left(\bar{a}_{i} \cdot V\right)\right],
$$

it is enough to show that $f^{-1}[W \cap(\bar{a} \cdot V)]$ is $\tau$-open whenever $\bar{a} \in G$.

Fix $\bar{a} \in G$. The set $W$ is $\tau$-open, so $\left(\bar{a}^{-1} \cdot W\right) \cap V$ is open in $V$. By Claim 2 , if $\bar{g}_{1}, \bar{g}_{2} \in G$, then the set

$$
\left.Z:=\left\{\langle\bar{x}, \bar{y}\rangle \in V \times V: \bar{a}^{-1} \cdot \bar{g}_{1}^{-1} \cdot \bar{x} \cdot \bar{g}_{2}^{-1} \cdot \bar{y} \in V\right\rangle\right\}
$$

is open in $V \times V$ and the map $F_{\bar{g}_{1}, \bar{g}_{2}}: Z \longrightarrow V$ defined by

$$
F_{\bar{g}_{1}, \bar{g}_{2}}(\bar{x}, \bar{y})=\bar{a}^{-1} \cdot \bar{g}_{1}^{-1} \cdot \bar{x} \cdot \bar{g}_{2}^{-1} \cdot \bar{y}
$$

is continuous. Hence $F_{\bar{g}_{1}, \bar{g}_{2}}^{-1}\left[\left(\bar{a}^{-1} \cdot W\right) \cap V\right]$ is open in $Z$, so in $V \times V$. Note that

$$
\begin{aligned}
F_{\bar{g}_{1}, \bar{g}_{2}}^{-1}\left[\left(\bar{a}^{-1} \cdot W\right) \cap V\right]= & \left\{\langle\bar{x}, \bar{y}\rangle \in V \times V: \bar{a}^{-1} \cdot \bar{g}_{1}^{-1} \cdot \bar{x} \cdot \bar{g}_{2}^{-1} \cdot \bar{y} \in\left(\bar{a}^{-1} \cdot W\right) \cap V\right\}= \\
& \left\{\left\langle\bar{g}_{1} \cdot \bar{x}, \bar{g}_{2} \cdot \bar{y}\right\rangle \in V \times V: \bar{x} \cdot \bar{y} \in W \cap(\bar{a} \cdot V)\right\}= \\
& \left(\left\langle\bar{g}_{1}, \bar{g}_{2}\right\rangle \cdot f^{-1}[W \cap(\bar{a} \cdot V)]\right) \cap(V \times V)
\end{aligned}
$$

whenever $\bar{g}_{1}, \bar{g}_{2} \in G$. Thus $f^{-1}[W \cap(\bar{a} \cdot V)]$ is $\tau$-open.

Modifying accordingly the proof of Theorem 3.5, we obtain the following variant of the above result.

Theorem 3.6 Assume that $\mathcal{M}$ is a first order topological structure with respect to some formula $\phi$ and $d: \operatorname{Def}(\mathcal{M}) \longrightarrow \mathbb{N} \cup\{-\infty\}$ is a dimension function. Assume also that $\mathcal{M}$ has the cell decomposition property and the continuity property with respect to $d$ and the topology determined by $\varphi$. If $(G, \cdot)$ is a group definable in $\mathcal{M}$ over $A$ with $G \subseteq M^{m}$ and $d(G)=n$, then there are an $A$-definable set $V \subseteq G$ and a topology $\tau$ on $G$ such that

(a) $(G, \cdot)$ with the topology $\tau$ is a topological group;

(b) $V$ is d-large in $G$ and open in $G$;

(c) if $n \geq 1$, then $V$ is a union of finitely many $\tau$-open A-definable pairwise disjoint sets which are A-definably homeomorphic with A-definable open subsets of $M^{n}$;

(d) the topology $\tau$ restricted to $V$ coincides with the topology of $V$ induced from $M^{m}$;

(e) some $n+1$ translates of $V$ cover $G$.

Under assumptions of Theorem 3.6, using the properties of the topology $\tau$ and the fact that finitely many translates of $V$ cover $G$, one can easily show that every definable subset of $G$ is a finite Boolean combination of $\tau$-open definable subsets of $G$ (or equivalently, a union of finitely many locally $\tau$-closed sets). Consequently using $\S 2$ of [Pi1], as in $\S 2$ [Pi2], one can show that any definable subgroup of $G$ is $\tau$-closed. 
It is quite clear that if $G$ is a group definable in a first order structure $\mathcal{M}$ equipped with a weak dimension function $d$ invariant under injective definable maps and $H$ is a definable subgroup of $G$ with $[G: H]$ finite, then $d(H)=d(G)$. Note that this implication in general cannot be reversed, a counterexample being a real closed field with a nontrivial valuation.

Groups definable in o-minimal structures satisfy the DCC on definable subgroups. This property in general is not satisfied by groups definable in weakly o-minimal structures as illustrated by the following example. Denote by $\mathcal{M}_{0}=(\mathbb{Q}, \leq,+)$ the ordered group of rationals and let for $i<\omega$, $\mathcal{M}_{i+1}$ be a proper elementary extension of $\mathcal{M}_{i}$ in which the type $\left\{0<x<a: a \in M_{i}, a>0\right\}$ is realized. Denote by $\mathcal{M}=(M, \leq,+)$ the direct limit of the elementary chain $\mathcal{M}_{0} \prec \mathcal{M}_{1} \prec \ldots$ and let

$$
P_{i}=\left\{x \in M:\left(\forall y \in M_{i} \backslash\{0\}\right)(|x|<|y|)\right\} .
$$

As previously, the structure $\mathcal{N}:=\left(M, \leq,+,\left(P_{i}\right)_{i<\omega}\right)$ has a weakly o-minimal theory and

$$
\left(P_{0},+\right)>\left(P_{1},+\right)>\left(P_{2},+\right), \ldots
$$

is a descending chain of groups definable in $\mathcal{N}$.

\section{Definable group actions}

Suppose that $\mathcal{M}=(M, \ldots)$ is a first order structure, $(G, \cdot)$ is a group definable in $\mathcal{M}$ [over $A \subseteq M]$ and $X \subseteq M^{r}$ is a non-empty set definable in $\mathcal{M}$ [over $A$ ]. A map $*: G \times X \longrightarrow X$ definable in $\mathcal{M}$ [over $A]$ is called a definable group action $[$ a group action definable over $A]$ iff $e_{G} * \bar{a}=\bar{a}$ and $\bar{g} *(\bar{h} * \bar{a})=(\bar{g} \cdot \bar{h}) * \bar{a}$ for any $\bar{g}, \bar{h} \in G$ and $\bar{a} \in X$. If $*$ is a group action of $(G, \cdot)$ on $X$, then in a natural way the group $G \times G$ acts on $G \times X$, and this action will also be denoted by $*$, i.e. $\left\langle\bar{g}_{1}, \bar{g}_{2}\right\rangle *\langle\bar{g}, \bar{a}\rangle=\left\langle\bar{g}_{1} \cdot \bar{g}, \bar{g}_{2} * \bar{a}\right\rangle$.

Lemma 4.1 Assume that $\mathcal{M}$ is a first order structure and $d: \operatorname{Def}(\mathcal{M}) \longrightarrow \mathbb{N} \cup\{-\infty\}$ is a function invariant under injective definable maps. Assume also that $*$ is a transitive definable group action of a group $(G, \cdot)$ on a set $X$. If $\bar{a}, \bar{b} \in X$, then

$$
d\left(G_{\bar{a}}\right)=d\left(G_{\bar{b}}\right)=d(\{\bar{g} \in G: \bar{g} * \bar{a}=\bar{b}\})=d\left(\left\{\bar{g} \in G: \bar{g}^{-1} * \bar{a}=\bar{b}\right\}\right) .
$$

Proof. If $\bar{a}, \bar{b} \in X, \bar{h} \in G$ and $\bar{h} * \bar{a}=\bar{b}$, then $G_{\bar{b}}=\bar{h} \cdot G_{\bar{a}} \cdot \bar{h}^{-1},\{\bar{g} \in G: \bar{g} * \bar{a}=\bar{b}\}=\bar{h} \cdot G_{\bar{a}}$ and $\left\{\bar{g} \in G: \bar{g}^{-1} * \bar{a}=\bar{b}\right\}=G_{\bar{a}} \cdot \bar{h}^{-1}$. The equality of all $d$-dimensions is a consequence of invariance of $d$ under injective definable maps.

Lemma 4.2 Assume that $\mathcal{M}$ is a first order structure and $d:$ Def $(\mathcal{M}) \longrightarrow \mathbb{N} \cup\{-\infty\}$ is a dimension function. Assume that $*$ is a transitive definable group action of a group $(G, \cdot)$ on a set $X$ definable in $\mathcal{M}$. If $\bar{a} \in X$, then

$$
d(G)=d(X)+d\left(G_{\bar{a}}\right) .
$$

Proof. Fix $\bar{a} \in X$ and define a surjection $f: G \longrightarrow X$ by setting $f(\bar{g})=\bar{g} * \bar{a}$. By Lemma 4.1, $d\left(f^{-1}(\bar{b})\right)=d\left(G_{\bar{a}}\right)$ whenever $\bar{b} \in X$. Since $G=\bigcup_{\bar{b} \in X} f^{-1}(\bar{b})$, by our assumption we are done.

Lemma 4.3 Assume that $\mathcal{M}$ is a first order structure and $d: \operatorname{Def}(\mathcal{M}) \longrightarrow \mathbb{N} \cup\{-\infty\}$ is a dimension function. Assume also that $*$ is a transitive definable group action of a group $(G, \cdot)$ on a set $X$ definable in $\mathcal{M}, U, W \subseteq X$ are definable sets, $W \neq \emptyset$, and $U$ is d-large in $X$. Then the set $\{\bar{g} \in G: \bar{g} * U$ is d-large in $W\}$ is d-large in $G$. 
Proof. Denote the $d$-dimensions of the sets $G, X, W, X \backslash U$ by $n, s, k$ and $l$ respectively. The case $U=X$ being trivial, let $U \neq X$. Our assumptions guarantee that $n \geq s>l \geq 0$ and $s \geq k \geq 0$. Consider the following set $S$ :

$$
S=\{\langle\bar{g}, \bar{a}\rangle \in G \times X: \bar{a} \in W \backslash(\bar{g} * U)\} .
$$

For $\bar{a} \in W$ let $f_{\bar{a}}: G \longrightarrow X$ be the map given by $f_{\bar{a}}(\bar{g})=\bar{g}^{-1} * \bar{a}$. If $\bar{a} \in W$ and $\bar{g} \in G$, then

$$
\begin{aligned}
S_{\bar{g}} & :=\{\bar{a} \in X:\langle\bar{g}, \bar{a}\rangle \in S\}=W \backslash(\bar{g} * U) ; \\
S^{\bar{a}}:=\{\bar{g} \in G:\langle\bar{g}, \bar{a}\rangle \in S\} & =f_{\bar{a}}^{-1}[X \backslash U] .
\end{aligned}
$$

Note that for $\bar{g} \in G$, the set $\bar{g} * U$ is not $d$-large in $W$ iff $d\left(S_{\bar{g}}\right)=k$. Moreover, if $\bar{a}, \bar{b} \in X, \bar{h} \in G$ and $\bar{h} * \bar{a}=\bar{b}$, then $f_{\bar{a}}^{-1}(\bar{b})=G_{\bar{a}} \cdot \bar{h}^{-1}$ and $d\left(f_{\bar{a}}^{-1}(\bar{b})\right)=d\left(G_{\bar{a}}\right)$. Consequently, by addition property of $d$ and Lemma 4.2 ,

$$
d\left(S^{\bar{a}}\right)=d\left(\bigcup_{\bar{b} \in X \backslash U} f_{\bar{a}^{-1}}(\bar{b})\right)=d(X \backslash U)+d\left(G_{\bar{a}}\right)=d(X \backslash U)+d(G)-d(X)=l+n-s
$$

whenever $\bar{a} \in W$. This implies that $d(S)=d(W)+l+n-s=k+l+n-s<k+n$.

Suppose for a contradiction that the set $\{\bar{g} \in G: \bar{g} * U$ is $d$-large in $W\}$ is not $d$-large in $G$. Then $d\left(\left\{\bar{g} \in G: d\left(S_{\bar{g}}\right)=k\right\}\right)=n$. The addition property together with (A4) imply that

$$
d(S) \geq d\left(\bigcup_{\left\{\bar{g} \in G: d\left(S_{\bar{g}}\right)=k\right\}}\{\bar{g}\} \times S_{\bar{g}}\right)=d\left(\left\{\bar{g} \in G: d\left(S_{\bar{g}}\right)=k\right\}\right)+k=n+k,
$$

which contradicts our previous calculations.

Lemma 4.4 Assume that $\mathcal{M}$ is a first order structure and $d: \operatorname{Def}(\mathcal{M}) \longrightarrow \mathbb{N} \cup\{-\infty\}$ is a dimension function. Assume also that $*$ is a transitive definable group action of a group $(G, \cdot)$ on a set $X$ definable in $\mathcal{M}$ and $U \subseteq X$ is a definable set, d-large in $X$. Then there are $\bar{g}_{0}, \ldots, \bar{g}_{s} \in G$ such that $X=\left(\bar{g}_{0} * U\right) \cup \ldots \cup\left(\bar{g}_{s} * U\right)$, where $s=d(X)$.

Proof. Using Lemma 4.3, we inductively find $\bar{g}_{0}, \ldots, \bar{g}_{s} \in G$ such that for every $i \leq s$,

$$
d\left(X \backslash\left(\left(\bar{g}_{0} * U\right) \cup \ldots \cup\left(\bar{g}_{i} * U\right)\right)\right)<s-i .
$$

For $i=s$ this means that $X=\left(\bar{g}_{0} * U\right) \cup \ldots \cup\left(\bar{g}_{s} * U\right)$.

Lemma 4.5 Assume that $\mathcal{M}$ is a first order structure and $d: \operatorname{Def}(\mathcal{M}) \longrightarrow \mathbb{N} \cup\{-\infty\}$ is a dimension function. Assume also that $*$ is a transitive definable group action of a group $(G, \cdot)$ on a set $X$ definable in $\mathcal{M}, V \subseteq G$ and $U \subseteq X$ are definable sets, $V$ is d-large in $G, U$ is d-large in $X$. Then for every $\bar{b} \in X$, the set $Y:=\{\langle\bar{g}, \bar{a}\rangle \in V \times U: \bar{g} * \bar{a}=\bar{b}\}$ has d-dimension equal to $d(G)$.

Proof. Fix $\bar{b} \in X$. By Lemma 4.3, the set $Z:=\{\bar{g} \in G: \bar{b} \in \bar{g} * U\}$ is $d$-large in $G$, so its intersection with $V$ is also $d$-large in $G$. Note that

$$
Y=\left\{\left\langle\bar{g}, \bar{g}^{-1} * \bar{b}\right\rangle \in G \times X: \bar{g} \in Z \cap V\right\} .
$$

Our assumptions guarantee that $d$ is invariant under injective definable maps, so $d(Y)=d(Z \cap V)=$ $d(G)$. 
Theorem 4.6 Assume that $\mathcal{M}$ is a first order topological structure with respect to some formula $\varphi(x ; \bar{y}), d: \operatorname{Def}(\mathcal{M}) \longrightarrow \mathbb{N} \cup\{-\infty\}$ is a dimension function and $\mathcal{M}$ has the continuity property with respect to $d$ and the topology determined by $\varphi$. Assume also that $A \subseteq M$ and $*: G \times X \longrightarrow X$ is a transitive $A$-definable group action of an $A$-definable group $(G, \cdot)$ on an $A$-definable set $X$ in M. There are A-definable sets $V \subseteq G$ and $U \subseteq X$, and topologies $\tau_{1}, \tau_{2}, \tau$ on $G, X$ and $G \times X$ respectively such that

(a) $\left(G, \cdot \tau_{1}\right)$ is a topological group;

(b) the set $V$ is d-large in $G$ and open in $G$, some $d(G)+1$ left (right) translates of $V$ cover $G, V=V^{-1}$, and the inversion map as well as the group operation are continuous functions with respect to $\tau_{1}$;

(c) the topology $\tau_{1}$ restricted to $V$ agrees with the topology of $V$ induced by the topology of $M$;

(d) the set $U$ is d-large in $X$, open in $X$, and $U=\left(\bar{g}_{0} * U\right) \cup \ldots \cup\left(\bar{g}_{s} * U\right)$, for some $\bar{g}_{0}, \ldots, \bar{g}_{s} \in G$ where $s=d(X)$;

(e) the topology $\tau_{2}$ restricted to $U$ agrees with the topology of $U$ induced by the topology of $M$;

(f) $\tau$ is the product topology determined by $\tau_{1}$ and $\tau_{2}$;

(g) the maps $\langle\bar{g}, \bar{a}\rangle \longmapsto \bar{g} * \bar{a}$ and $\langle\bar{g}, \bar{a}\rangle \longmapsto \bar{g}^{-1} * \bar{a}$ are continuous with respect to the topologies $\tau$ and $\tau_{2}$

(h) if $\bar{a} \in X$, then the maps $\bar{g} \longmapsto \bar{g} * \bar{a}$ and $\bar{g} \longmapsto \bar{g}^{-1} * \bar{a}$ are continuous surjections from $G$ to $X$ with respect to the topologies $\tau_{1}$ and $\tau_{2}$; if additionally $*$ is faithful, then they are homeomorphisms from $G$ onto $X$;

(i) if $\bar{g} \in G$, then the map $\bar{a} \longmapsto \bar{g} * \bar{a}$ is a homeomorphism from $X$ onto $X$ with respect to the topology $\tau_{2}$.

Proof. The existence of an $A$-definable set $V \subseteq G$ and a topology $\tau_{1}$ on $G$ for which the conditions (a)-(c) are satisfied is guaranteed by Theorem 3.5 and its proof. Moreover (by the prof of Theorem $3.5)$, there exists an $A$-definable set $Y \subseteq V \times V$ such that $Y$ is open in $G \times G$ and $d$-large in $G \times G$, the group operation - restricted to $Y$ is continuous and for every $\bar{a} \in V$, the sets

$$
\{\bar{b} \in G:\langle\bar{b}, \bar{a}\rangle \in Y\},\left\{\bar{b} \in G:\left\langle\bar{b}^{-1}, \bar{b} \cdot \bar{a}\right\rangle \in Y\right\}
$$

are $d$-large in $G$.

Denote the group action $*$ by $F$ (i.e. $F(\bar{g}, \bar{a})=\bar{g} * \bar{a}$ whenever $\bar{g} \in G$ and $\bar{a} \in X$ ). There is an $A$-definable set $Y_{0} \subseteq G \times X$ such that $Y_{0}$ is open in $G \times X, d$-large in $G \times X$ and $F$ restricted to $Y_{0}$ is continuous. Let

$$
\begin{aligned}
& W_{1}=\left\{\bar{a} \in X:\left\{\bar{g} \in G:\langle\bar{g}, \bar{a}\rangle \in Y_{0}\right\} \text { is d-large in } G\right\} \\
& W_{2}=\left\{\bar{a} \in X:\left\{\bar{g} \in G:\left\langle\bar{g}^{-1}, \bar{g} * \bar{a}\right\rangle \in Y_{0}\right\} \text { is d-large in } G\right\} .
\end{aligned}
$$

The sets $W_{1}, W_{2}$ are both $A$-definable and reasoning as in the proof of Theorem 3.5 shows that they are both $d$-large in $X$. There exists an $A$-definable set $U \subseteq W_{1} \cap W_{2}$ which is open in $X$ and $d$-large in $X$. The remaining part of $(\mathrm{d})$ is a consequence of Lemma 4.4. As in the prof of Theorem 3.5 , we get the following claim.

Claim 1. (a) For any $\bar{g} \in G$ and $\bar{a} \in X$, the set

$$
Z(\bar{g}, \bar{a}):=\{\bar{x} \in V:(\bar{g} \cdot \bar{x}) * \bar{a} \in U\}
$$

is open in $V$ and the function $\bar{x} \longmapsto(\bar{g} \cdot \bar{x}) * \bar{a}$ is a continuous map from $Z(\bar{g}, \bar{a})$ onto $(\bar{g} \cdot Z(\bar{g}, \bar{a})) * \bar{a}$. 
(b) For any $\bar{g}, \bar{h} \in G$, the set

$$
Z^{\prime}(\bar{g}, \bar{h}):=\{\langle\bar{x}, \bar{y}\rangle \in V \times U:(\bar{g} \cdot \bar{x} \cdot \bar{h}) * \bar{y} \in U\}
$$

is open in $V \times U$, and the function $\langle\bar{x}, \bar{y}\rangle \longmapsto(\bar{g} \cdot \bar{x} \cdot \bar{h}) * \bar{y}$ is a continuous map from $Z^{\prime}(\bar{g}, \bar{h})$ to $U$.

Define the topology $\tau_{2}$ on $X$ as follows: a set $Z \subseteq X$ is $\tau_{2}$-open iff for any $\bar{g} \in G$, the set $(\bar{g} * Z) \cap U$ is open in $U$. Clearly, if $Z \subseteq U$, then $Z$ is $\tau_{2}$-open iff $Z$ is open in $U$. Let $\tau$ be the product topology on $G \times X$ determined by $\tau_{1}$ and $\tau_{2}$.

Claim 2. A definable set $Z \subseteq G \times X$ is $\tau$-open iff

$$
\left(\forall \bar{g}_{1}, \bar{g}_{2} \in G\right)\left(\left(\left\langle\bar{g}_{1}, \bar{g}_{2}\right\rangle * Z\right) \cap Y \text { is } \tau \text {-open in } G \times X\right) .
$$

Proof of Claim 2. For the left-to-right implication assume that $Z \subseteq G \times X$ is a $\tau$-open set, $\bar{g}_{1}, \bar{g}_{2} \in G$, and $\langle\bar{g}, \bar{a}\rangle \in\left(\left\langle\bar{g}_{1}, \bar{g}_{2}\right\rangle * Z\right) \cap Y$. Since $Y$ is open in $G \times X$, there are $V_{1} \subseteq G$ and $U_{1} \subseteq X$ such that $V_{1}$ is open in $G, U_{1}$ is open in $X$ and

$$
\langle\bar{g}, \bar{a}\rangle \in V_{1} \times U_{1} \subseteq Y \subseteq V \times U .
$$

Note that

$$
\left\langle\bar{g}_{1}^{-1} \cdot \bar{g}, \bar{g}_{2}^{-1} * \bar{a}\right\rangle \in Z \cap\left(\left\langle\bar{g}_{1}^{-1}, \bar{g}_{2}^{-1}\right\rangle * Y\right) .
$$

Since $Z$ is $\tau$-open, there are $V_{2} \subseteq G$ and $U_{2} \subseteq X$ such that $V_{2}$ is $\tau_{1}$-open, $U_{2}$ is $\tau_{2}$-open and

$$
\left\langle\bar{g}_{1}^{-1} \cdot \bar{g}, \bar{g}_{2}^{-1} * \bar{a}\right\rangle \in V_{2} \times U_{2} \subseteq Z .
$$

It is clear that the sets $\left(\bar{g}_{1} \cdot V_{2}\right) \cap V$ and $\left(\bar{g}_{2} * U_{2}\right) \cap U$ are open in $G$ and $X$ respectively, and

$$
\langle\bar{g}, \bar{a}\rangle \in\left(\left(\bar{g}_{1} \cdot V_{2}\right) \cap V\right) \times\left(\left(\bar{g}_{2} * U_{2}\right) \cap U\right) \subseteq\left\langle\bar{g}_{1}, \bar{g}_{2}\right\rangle * Z .
$$

In this way we have shown that the set $\left(\left\langle\bar{g}_{1}, \bar{g}_{2}\right\rangle * Z\right) \cap Y$ is $\tau$-open.

For the right-to-left direction assume that $Z \subseteq G \times X$,

$$
\left(\forall \bar{g}_{1}, \bar{g}_{2} \in G\right)\left(\left(\left\langle\bar{g}_{1}, \bar{g}_{2}\right\rangle * Z\right) \cap Y \text { is } \tau \text {-open in } G \times X\right),
$$

and $\langle\bar{g}, \bar{a}\rangle \in Z$. There are $\bar{g}_{1}, \bar{g}_{2} \in G$ such that

$$
\left\langle\bar{g}_{1} \cdot \bar{g}, \bar{g}_{2} * \bar{a}\right\rangle \in\left(\left\langle\bar{g}_{1}, \bar{g}_{2}\right\rangle * Z\right) \cap Y \subseteq V \times U .
$$

So there are sets $U_{1} \subseteq X$ and $V_{1} \subseteq G$ such that $U_{1}$ is open in $X, V_{1}$ is open in $G$ and

$$
\left\langle\bar{g}_{1} \cdot \bar{g}, \bar{g}_{2} * \bar{a}\right\rangle \in V_{1} \times U_{1} \subseteq\left(\left\langle\bar{g}_{1}, \bar{g}_{2}\right\rangle * Z\right) \cap Y .
$$

Then $\bar{g}_{1}^{-1} \cdot V_{1}$ is $\tau_{1}$-open, $\bar{g}_{2}^{-1} * U_{1}$ is $\tau_{2}$-open, and

$$
\langle\bar{g}, \bar{a}\rangle \in\left(\bar{g}_{1}^{-1} \cdot V_{1}\right) \times\left(\bar{g}_{2}^{-1} * U_{1}\right) \subseteq Z .
$$

So $Z$ is $\tau$-open.

Claim 3. The maps $\langle\bar{g}, \bar{a}\rangle \longmapsto \bar{g} * \bar{a}$ and $\langle\bar{g}, \bar{a}\rangle \longmapsto \bar{g}^{-1} * \bar{a}$ are continuous with respect to the topologies $\tau$ and $\tau_{2}$. 
Proof of Claim 3. Let $W \subseteq X$ be a $\tau_{2}$-open set. We will be done if we prove that $F^{-1}[W]$ is $\tau$-open. Of course it is enough to show that the set $F^{-1}[W \cap(\bar{g} * U)]$ is $\tau$-open whenever $\bar{g} \in G$.

Fix $\bar{g} \in G$. The set $W$ is $\tau$-open, so $\left(\bar{g}^{-1} * W\right) \cap U$ is open in $X$. By Claim 1 (b), the set

$$
Z:=\left\{\langle\bar{x}, \bar{y}\rangle \in V \times U:\left(\bar{g}^{-1} \cdot \bar{g}_{1}^{-1} \cdot \bar{x} \cdot \bar{g}_{2}^{-1}\right) * \bar{y} \in U\right\}
$$

is open in $V \times U$ and the map $F_{\bar{g}_{1}, \bar{g}_{2}}: Z \longrightarrow U$ defined by $F_{\bar{g}_{1}, \bar{g}_{2}}(\bar{x}, \bar{y})=\left(\bar{g}^{-1} \cdot \bar{g}_{1}^{-1} \cdot \bar{x} \cdot \bar{g}_{2}^{-1}\right) * \bar{y}$ is continuous. Hence the set $F_{\bar{g}_{1}, \bar{g}_{2}}^{-1}\left[\left(\bar{g}^{-1} \cdot W\right) \cap U\right]$ is open in $G \times X$. Note that

$$
\begin{aligned}
F_{\bar{g}_{1}, \bar{g}_{2}}^{-1}\left[\left(\bar{g}^{-1} \cdot W\right) \cap U\right]= & \left\{\langle\bar{x}, \bar{y}\rangle \in V \times U:\left(\bar{g}^{-1} \cdot \bar{g}_{1}^{-1} \cdot \bar{x} \cdot \bar{g}_{2}^{-1}\right) * \bar{y} \in\left(\bar{g}^{-1} * W\right) \cap U\right\}= \\
& \left\{\left\langle\bar{g}_{1} \cdot \bar{x}, \bar{g}_{2} * \bar{y}\right\rangle \in V \times U: \bar{x} * \bar{y} \in W \cap(\bar{g} * U)\right\}= \\
& \left(\left\langle\bar{g}_{1}, \bar{g}_{2}\right\rangle * F^{-1}[W \cap(\bar{g} * U)]\right) \cap(V \times U)
\end{aligned}
$$

whenever $\bar{g}_{1}, \bar{g}_{2} \in G$. Thus by Claim 2 , the set $F^{-1}[W \cap(\bar{g} * U)]$ is $\tau$-open.

The above argument and the fact that the inversion map is $\tau_{1}$-continuous on $G$ imply that the map $\langle\bar{g}, \bar{a}\rangle \longmapsto \bar{g}^{-1} * \bar{a}$ is continuous with respect to the topologies $\tau$ and $\tau_{2}$.

(h) and (i) are easy consequences of (g). Alternatively, one could use Claim 1(a) to show that the map $\bar{g} \longmapsto \bar{g}^{-1} * \bar{a}$ is continuous with respect to the topologies $\tau_{1}$ and $\tau_{2}$.

\section{Definable fields}

Let $\mathcal{M}=(M, \ldots)$ is a first order structure and $A \subseteq M$. A field $(K,+, \cdot)$ is said to be definable in $\mathcal{M}$ [over $A$ ] iff there is a positive integer $m$ such that $K \subseteq M^{m}$ is a definable [over $A$ ] set and there are definable [over $A$ ] sets $X, Y \subseteq K^{3} \subseteq M^{3 m}$ such that for any $\bar{a}, \bar{b}, \bar{c} \in K$,

$$
\langle\bar{a}, \bar{b}, \bar{c}\rangle \in X \text { iff } \bar{a}+\bar{b}=\bar{c} \text {, and }\langle\bar{a}, \bar{b}, \bar{c}\rangle \in Y \text { iff } \bar{a} \cdot \bar{b}=\bar{c} .
$$

It is easy to see that if $K$ is a field definable in $\mathcal{M}$ and $L$ is its finite extension, then $L$ is isomorphic to a field definable in $\mathcal{M}$.

Theorem 5.1 Assume that $\mathcal{M}=(M, \ldots)$ is a first order topological structure with respect to some formula $\varphi(x ; \bar{y}), d: \operatorname{Def}(\mathcal{M}) \longrightarrow \mathbb{N} \cup\{-\infty\}$ is a dimension function and $\mathcal{M}$ has the continuity property with respect to $d$ and the topology determined by $\varphi$. Assume also that $(K,+, \cdot)$ is a field definable in $\mathcal{M}$ over $A$ with $K \subseteq M^{m}$ and $d(K)=n$. Then there are an $A$-definable set $V \subseteq K$ and a topology $\tau$ on $K$ such that

(a) $(K,+, \cdot)$ with the topology $\tau$ is a topological field;

(b) $V$ is d-large in $K$ and open in $K$;

(c) the topology $\tau$ restricted to $V$ coincides with the topology of $V$ induced from $M^{m}$;

(d) some $n+1$ additive translates of $V$ cover $K$ and some $n+1$ multiplicative translates of $V$ cover $K^{*}$.

Proof. There exists an $A$-definable set $V_{0} \subseteq K$ such that $0_{K} \notin V_{0}, V_{0}$ is open in $K$ and $d$-large in $K$, and both the additive and multiplicative inverses restricted to $V_{0}$ are continuous. By the continuity property, there is an $A$-definable set

$$
V_{1} \subseteq V_{0} \cap\left(-V_{0}\right) \cap V_{0}^{-1} \cap\left(-V_{0}^{-1}\right)
$$


such that $V_{1}$ is open in $K$ and $d$-large in $K$. Both the additive and multiplicative inversions restricted to $V_{1}$ are continuous maps from $V_{1}$ to $V_{0}$. Denote the addition and multiplication by $f$ and $g$ respectively. It is easy to see that the set $\left(V_{0} \times V_{0}\right) \cap f^{-1}\left[V_{0}\right] \cap g^{-1}\left[V_{0}\right]$ is $A$-definable and $d$-large in $K \times K$. Hence there exists an $A$-definable set $Y_{0} \subseteq\left(V_{0} \times V_{0}\right) \cap f^{-1}\left[V_{0}\right] \cap g^{-1}\left[V_{0}\right]$ which is open in $K \times K$ and $d$-large in $K \times K$. Moreover, $f \uparrow Y_{0} g \uparrow Y_{0}$ are continuous maps from $Y_{0}$ to $V_{0}$. Now define

$$
\begin{aligned}
& W_{1}=\left\{\bar{a} \in V_{1}:\left\{\bar{b} \in K:\langle\bar{b}, \bar{a}\rangle \in Y_{0}\right\} \text { is d-large in } K\right\} \\
& W_{2}=\left\{\bar{a} \in V_{1}:\left\{\bar{b} \in K:\langle-\bar{b}, \bar{b}+\bar{a}\rangle \in Y_{0}\right\} \text { is } d \text {-large in } K\right\} \\
& W_{3}=\left\{\bar{a} \in V_{1}:\left\{\bar{b} \in K^{*}:\left\langle\bar{b}^{-1}, \bar{b} \cdot \bar{a}\right\rangle \in Y_{0}\right\} \text { is d-large in } K\right\} .
\end{aligned}
$$

Repeating a suitable argument from the proof of Theorem 3.5, we see that the set $V_{1}^{\prime}:=W_{1} \cap W_{2} \cap$ $W_{3}$ is $A$-definable and $d$-large in $K$. There is an $A$-definable set $V_{2} \subseteq V_{1}^{\prime}$ such that $V_{2}$ is open in $K$ and $d$-large in $K$. Let

$$
V=V_{2} \cap\left(-V_{2}\right) \cap V_{2}^{-1} \cap\left(-V_{2}^{-1}\right) .
$$

$V$ is $A$-definable, open in $K$ and $d$-large in $K$. Moreover, $V=-V=V^{-1}=-V^{-1}$. Also $V \times V$ is $A$-definable, open in $K \times K$ and $d$-large in $K \times K$. Let

$$
Y=(V \times V) \cap Y_{0} \cap f^{-1}[V] \cap g^{-1}[V] .
$$

The set $Y$ is $A$-definable, open in $K \times K$ and $d$-large in $K \times K$. Addition and multiplication restricted to $Y$ are continuous maps from $Y$ to $V$. Moreover, if $\bar{a} \in V$, then the sets

$$
\{\bar{b} \in K:\langle\bar{b}, \bar{a}\rangle \in Y\},\{\bar{b} \in K:\langle-\bar{b}, \bar{b}+\bar{a}\rangle \in Y\},\left\{\bar{b} \in K:\left\langle\bar{b}^{-1}, \bar{b} \cdot \bar{a}\right\rangle \in Y\right\}
$$

are all $A$-definable and $d$-large in $K$. Define the topology $\tau$ on $K$ as follows: $X \subseteq K$ is $\tau$-open iff for all $\bar{k} \in K,(\bar{k}+X) \cap V$ is open in $V$. Clearly, the topology $\tau$ restricted to $V$ agrees with topology topology of $V$ induced from $M^{m}$. By the proof of Theorem $3.5,(K,+, \tau)$ is a topological group.

Claim 1. (a) If $\bar{a} \in K$, then the set $X(\bar{a}):=\{\bar{x} \in V: \bar{a} \cdot \bar{x} \in V\}$ is open in $V$ and the function $\bar{x} \longmapsto \bar{a} \cdot \bar{x}$ is a continuous map from $X(\bar{a})$ to $V$. If $\bar{a} \neq 0_{K}$, then this map is a homeomorphism from $X$ onto $\bar{a} \cdot X$.

(b) If $\bar{a}, \bar{b}, \bar{c} \in K$, then the set

$$
Z(\bar{a}, \bar{b}, \bar{c}):=\{\langle\bar{x}, \bar{y}\rangle \in V \times V: \bar{c}+\bar{a} \cdot \bar{x}+\bar{b} \cdot \bar{y}+\bar{x} \cdot \bar{y} \in V\}
$$

is open in $V$, and the function $\langle\bar{x}, \bar{y}\rangle \longmapsto \bar{c}+\bar{a} \cdot \bar{x}+\bar{b} \cdot \bar{y}+\bar{x} \cdot \bar{y}$ is a continuous map from $Z(\bar{a}, \bar{b}, \bar{c})$ to $V$.

Proof of Claim 1. (a) is proved as Claim 1 in the proof of Theorem 3.5. For the proof of (b), fix $\bar{a}, \bar{b}, \bar{c} \in K$. We will show that for every $\left\langle\bar{x}_{0}, \bar{y}_{0}\right\rangle \in Z(\bar{a}, \bar{b}, \bar{c})$, there exists a subset of $Z(\bar{a}, \bar{b}, \bar{c})$ containing $\left\langle\bar{x}_{0}, \bar{y}_{0}\right\rangle$ which is open in $V \times V$ and on which the map

$$
\langle\bar{x}, \bar{y}\rangle \longmapsto \bar{c}+\bar{a} \cdot \bar{x}+\bar{b} \cdot \bar{y}+\bar{x} \cdot \bar{y}
$$

is continuous. This is sufficient. 
Fix $\left\langle\bar{x}_{0}, \bar{y}_{0}\right\rangle \in Z(\bar{a}, \bar{b}, \bar{c})$ and define

$$
\begin{aligned}
Z_{0}= & \left\{\bar{d} \in V:\left\langle\bar{d} \cdot \bar{a}, \bar{x}_{0}\right\rangle \in Y,\left\langle\bar{d} \cdot \bar{b}, \bar{y}_{0}\right\rangle \in Y,\left\langle\bar{d}, \bar{x}_{0}\right\rangle \in Y,\left\langle d \cdot \bar{x}_{0}, \bar{y}_{0}\right\rangle \in Y,\right. \\
& \left.\bar{d} \cdot\left(\bar{c}+\bar{a} \cdot \bar{x}_{0}+\bar{b} \cdot \bar{y}_{0}+\bar{x} \cdot \bar{y}\right) \in V,\left\langle\bar{d}^{-1}, \bar{d} \cdot\left(\bar{c}+\bar{a} \cdot \bar{x}_{0}+\bar{b} \cdot \bar{y}_{0}+\bar{x}_{0} \cdot \bar{y}_{0}\right)\right\rangle \in Y\right\} .
\end{aligned}
$$

The set $Z_{0}$ is large in $K$ so it is nonempty. Fix $\bar{d} \in Z_{0}$ and let

$$
\begin{aligned}
Z_{1}= & \{\langle\bar{x}, \bar{y}\rangle \in V \times V:\langle\bar{d} \cdot \bar{a}, \bar{x}\rangle \in Y,\langle\bar{d} \cdot \bar{b}, \bar{y}\rangle \in Y,\langle\bar{d}, \bar{x}\rangle \in Y,\langle d \cdot \bar{x}, \bar{y}\rangle \in Y, \\
& \left.\bar{d} \cdot(\bar{c}+\bar{a} \cdot \bar{x}+\bar{b} \cdot \bar{y}+\bar{x} \cdot \bar{y}) \in V,\left\langle\bar{d}^{-1}, \bar{d} \cdot(\bar{c}+\bar{a} \cdot \bar{x}+\bar{b} \cdot \bar{y}+\bar{x} \cdot \bar{y})\right\rangle \in Y\right\} .
\end{aligned}
$$

Obviously, $\left\langle\bar{x}_{0}, \bar{y}_{0}\right\rangle \in Z_{1} \subseteq Z(\bar{a}, \bar{b}, \bar{c})$. The set $Z_{1}$ is open in $V \times V$ and the function

$$
\langle\bar{x}, \bar{y}\rangle \longmapsto \bar{c}+\bar{a} \cdot \bar{x}+\bar{b} \cdot \bar{y}+\bar{x} \cdot \bar{y}
$$

restricted to $Z_{1}$ is a continuous map from $Z_{1}$ to $V$.

Claim 2. (a) Multiplication is a $\tau$-continuous map from $K \times K$ to $K$.

(b) Multiplicative inversion is a $\tau$-homeomorphism from $K^{*}$ to $K^{*}$.

Proof of Claim 2. (a) Let $W \subseteq K$ be a $\tau$-open set. We will be done if we show that the set $g^{-1}[W]$ is $\tau$-open. By Lemma 2.5, there are $\bar{c}_{0}, \ldots, \bar{c}_{n} \in K$ such that $K=\left(\bar{c}_{0}+V\right) \cup \ldots \cup\left(\bar{c}_{n}+V\right)$. Since

$$
g^{-1}[W]=g^{-1}\left[W \cap\left(\left(\bar{c}_{0}+V\right) \cup \ldots \cup\left(\bar{c}_{n}+V\right)\right)\right]=\bigcup_{i \leq n} g^{-1}\left[W \cap\left(\bar{c}_{i}+V\right)\right],
$$

it is enough to show that $g^{-1}[W \cap(\bar{c}+V)]$ is $\tau$-open whenever $\bar{c} \in K$. So fix $\bar{c} \in K$. By assumption, $(-\bar{c}+W) \cap V$ is open in $V$. Claim 1 (b) implies that for any $\bar{a}, \bar{b} \in K$, the set

$$
\begin{gathered}
\{\langle\bar{x}, \bar{y}\rangle \in V \times V:(-\bar{c}+\bar{a} \cdot \bar{b})-\bar{b} \cdot \bar{x}-\bar{a} \cdot \bar{y}+\bar{x} \cdot \bar{y} \in(-\bar{c}+W) \cap V\}= \\
\left(\langle\bar{a}, \bar{b}\rangle+g^{-1}[W \cap(\bar{c}+V)]\right) \cap(V \times V)
\end{gathered}
$$

is open in $V \times V$. This means that the set $g^{-1}[W \cap(\bar{c}+V)]$ is $\tau$-open.

The proof of (b) is similar to the proof of Claim 3 in the proof of Theorem 3.5 .

The proof of the following theorem is a rather straightforward modification of the above proof of Theorem 5.1.

Theorem 5.2 Assume that $\mathcal{M}$ is a first order topological structure with respect to some formula $\phi(x ; \bar{y})$ and $d: \operatorname{Def}(\mathcal{M}) \longrightarrow \mathbb{N} \cup\{-\infty\}$ is a dimension function. Assume also that $\mathcal{M}$ has the cell decomposition property and the continuity property with respect to $d$ and the topology determined by $\varphi$. If $(K,+, \cdot)$ is a field definable in $\mathcal{M}$ over $A$ with $K \subseteq M^{m}$ and $d(K)=n$, then there exist an $A$-definable set $V \subseteq K$ and a topology $\tau$ on $K$ such that

(a) $(K,+, \cdot)$ with the topology $\tau$ is a topological field;

(b) $V$ is d-large in $K$ and open in $K$;

(c) if $n \geq 1$, then $V$ is a union of finitely many $\tau$-open A-definable pairwise disjoint sets which are A-definably homeomorphic with A-definable open subsets of $M^{n}$;

(d) the topology $\tau$ restricted to $V$ coincides with the topology of $V$ induced from $M^{m}$;

(e) some $n+1$ additive translates of $V$ cover $K$ and some $n+1$ multiplicative translates of $V$ cover $K^{*}$. 


\section{References}

[vdD1] L. van den Dries, Dimension of Definable Sets, Algebraic Boundedness and Henselian Fields, Ann. Pure Appl. Logic 45 (1989), 189-209.

[vdD2] L. van den Dries, Tame Topology and o-minimal Structures, London Mathematical Society Lecture Notes Series, vol. 248, Cambridge: Cambridge University Press 1998.

[Ma1] L. Matthews, Prime models and t-minimal structures, preprint, Oxford University, 1992.

[Ma2] L. Matthews, Cell decomposition and dimension functions in first-order topological structures, Proc. London Math. Soc. 70 (1995), 1-32.

[MMS] D. Macpherson, D. Marker, and C. Steinhorn, Weakly o-minimal structures and real closed fields, Trans. Amer. Math. Soc. 352 (2000), 5435-5483.

[Mo] A. Mosley, Groups Definable in Topological Structures, PhD thesis, Queen Mary and Westfield College, London University, 1996.

[Pi1] A. Pillay, First order topological structures and theories, J. Symbolic Logic 52 (1987), 763-778.

[Pi2] A. Pillay, On groups and fields definable in o-minimal structures, J. Pure Appl. Algebra 53 (1988), 239-255.

[W] A. Weil, On algebraic groups of transformation, Amer. J. Math. 77 (1955), 355-391.

[We06] R. Wencel, Topological properties of sets definable in weakly o-minimal structures, J. Symbolic Logic 75 (2010), 841-867.

[We07] R. Wencel, Weakly o-minimal non-valuational structures. Annals of Pure and Applied Logic 154 (2008), 139-162.

Mailing adress : Instytut Matematyczny Uniwersytetu Wrocławskiego pl. Grunwaldzki 2/4, 50-384 Wrocław, POLAND

E-mail: $\quad$ rwenc@math.uni.wroc.pl 